\title{
Adoption of Vehicular Ad Hoc Networking Protocols by Networked Robots
}

\author{
Wim Vandenberghe · Ingrid Moerman · Piet \\ Demeester
}

Received: date / Accepted: date

\begin{abstract}
This paper focuses on the utilization of wireless networking in the robotics domain. Many researchers have already equipped their robots with wireless communication capabilities, stimulated by the observation that multi-robot systems tend to have several advantages over their single-robot counterparts. Typically, this integration of wireless communication is tackled in a quite pragmatic manner, only a few authors presented novel Robotic Ad Hoc Network (RANET) protocols that were designed specifically with robotic use cases in mind. This is in sharp contrast with the domain of vehicular ad hoc networks (VANET). This observation is the starting point of this paper. If the results of previous efforts focusing on VANET protocols could be reused in the RANET domain, this could lead to rapid progress in the field of networked robots. To investigate this possibility, this paper provides a thorough overview of the related work in the domain of robotic and vehicular ad hoc networks. Based on this information, an exhaustive list of requirements is defined for both types. It is concluded that the most significant difference lies in the fact that VANET protocols are oriented towards low throughput messaging, while RANET protocols have to support high throughput media streaming as well. Although not always with equal importance, all other defined requirements are valid for both protocols. This leads to the conclusion that cross-fertilization between them is an appealing approach for future RANET research. To support such developments, this paper concludes with the definition of an appropriate working plan.
\end{abstract}

Keywords Robotic Ad Hoc Network - Vehicular Ad Hoc Network · Networked robot $\cdot$ Wireless robot $\cdot$ V2V $\cdot$ V2I

W. Vandenberghe $\cdot$ I. Moerman $\cdot$ P. Demeester

Ghent University - IBBT

Department of Information Technology (INTEC)

Gaston Crommenlaan 8 bus 201, 9050 Ghent, Belgium

Tel.: +3293314900

Fax: +3293314899

E-mail: wim.vandenberghe@intec.ugent.be 


\section{Introduction}

In the last decade, a tremendous amount of technical developments have lead to significant progression in the domain of robotics. Robots have been applied successfully in a large number of diverge use cases such as rescue operations, fire fighting, underground mining, exploration, robot sports, etc. Key driver behind these scientific advancements is the integration of several fields such as mechanics, sensor systems, artificial intelligence, ubiquitous computing, wireless networking, and so on. In this paper we focus on the application of wireless networking in the robotics domain. Many researchers have already equipped their robots with wireless communication capabilities, stimulated by the observation that multi-robot systems tend to have several advantages over their single-robot counterparts. A team of robots can handle a wider range of tasks and accomplish some tasks more efficiently than a single robot. An example is path finding in a random maze composed of walls and paths, where cooperation results in significant better performance [1]. Teams of robots can also be beneficial in terms of reliability and scalability [2]. Reliability means that a failure in some robots does not seriously affect the system performance itself. Scalability means that the system is applicable to a wide variety of scenario's and environments for an envisaged application. Using teams of robots also greatly enhances the supported distance between a tele-operated robot and the human operator in dangerous situations [3]. Finally, in several cases the cost of building many simple robots is also significantly lower than the cost of a large and complex monolithic robot [4].

Typically, this integration of wireless communication is tackled in a quite pragmatic manner. In a large amount of published studies, the robots are equipped with IEEE 802.11 technology, in combination with an existing Mobile Ad Hoc Network (MANET) protocol. Such a protocol enables the robots to form an ad hoc network. There are no fixed routers in such a network, all nodes are capable of movement and can be connected dynamically in an arbitrary manner. Nodes of these networks function as routers which discover and maintain routes to other nodes in the network [5]. In some cases, these existing MANET protocols are enhanced to comply better with specific requirements of the studied robotic application. Such protocols are also called known as Robotic Ad Hoc Networks (RANET). However, only a few authors presented novel RANET protocols that were designed from scratch with the robotic use case in mind.

This is in sharp contrast with the domain of vehicular ad hoc networks (VANET). Such networks support vehicle-to-vehicle (V2V) and vehicle-to-infrastructure (V2I) communication to increase the "time horizon", the quality and reliability of information available to drivers. In research, this technology is applied in a large number of applications. When approaching dangerous situations such as a the tail of a traffic jam, an obstacle on the road or a ghost driver, drivers can be warned in time to avoid collision. Detected hazardous road conditions such as black ice or an oil trail can be automatically communicated. Drivers can be notified well in advance about approaching emergency vehicles, and can be directed to yield way in a uniform manner. This is just a small selection from the large number of applications that are made possible, but it is obvious that these systems can make a significant positive contribution to traffic safety. Attracted by this important potential, numerous research efforts 
developed novel VANET protocols, focusing on aspects such as high mobility, scalability and latency.

This observation that the field of VANET protocols is quite mature in comparison with the field of RANET protocols is the starting point of this paper. If the results of all previous efforts focusing on VANET protocols could be reused in the RANET domain, this could lead to rapid progress in the field of networked robots. To investigate this possibility, this paper provides a thorough overview of the state of the art in the domain of networked robots in section 2. This survey aims to illustrate the level of matureness of the domain, and to define the requirements of a RANET. In section 3 the same approach is followed for networked vehicles. In section 4, the two domains are compared and recommendations are specified to transfer results from the VANET to the RANET domain. Final conclusions are drawn at the end of the paper.

\section{Networked robots}

This section elaborates on different aspects of networked robots. A good starting point for this discussion is to provide a definition of such robots. In this paper, we rely on the definition provided by the IEEE Society of Robotics and Automation's Technical Committee on Networked Robots, since it is both clear and thorough [6]:

"A 'networked robot' is a robotic device connected to a communications network such as the Internet or LAN. The network could be wired or wireless, and based on any of a variety of protocols such as TCP, UDP or 802.11. Many new applications are now being developed ranging from automation to exploration. There are two subclasses of Networked Robots: (1) Tele-operated, where human supervisors send commands and receive feedback via the network. Such systems support research, education and public awareness by making valuable resources accessible to broad audiences; (2) Autonomous, where robots and sensors exchange data via the network. In such systems, the sensor network extends the effective sensing range of the robots, allowing them to communicate with each other over long distances to coordinate their activity. The robots in turn can deploy, repair and maintain the sensor network to increase its longevity, and utility. A broad challenge is to enable such new capabilities."

This definition indicates that networked robots can be deployed for a large number of various applications, leading to quite diverse RANET communication patterns in terms of network topology and data types. The next subsection presents a survey of these applications and the existing RANET solutions that support them. The idea is that the insights provided by such an investigation of the related work will result in a solid foundation for the definition of the communication requirements for networked robots. In section 4 they will be applied to assess the similarities between the requirements of RANET and VANET protocols. 

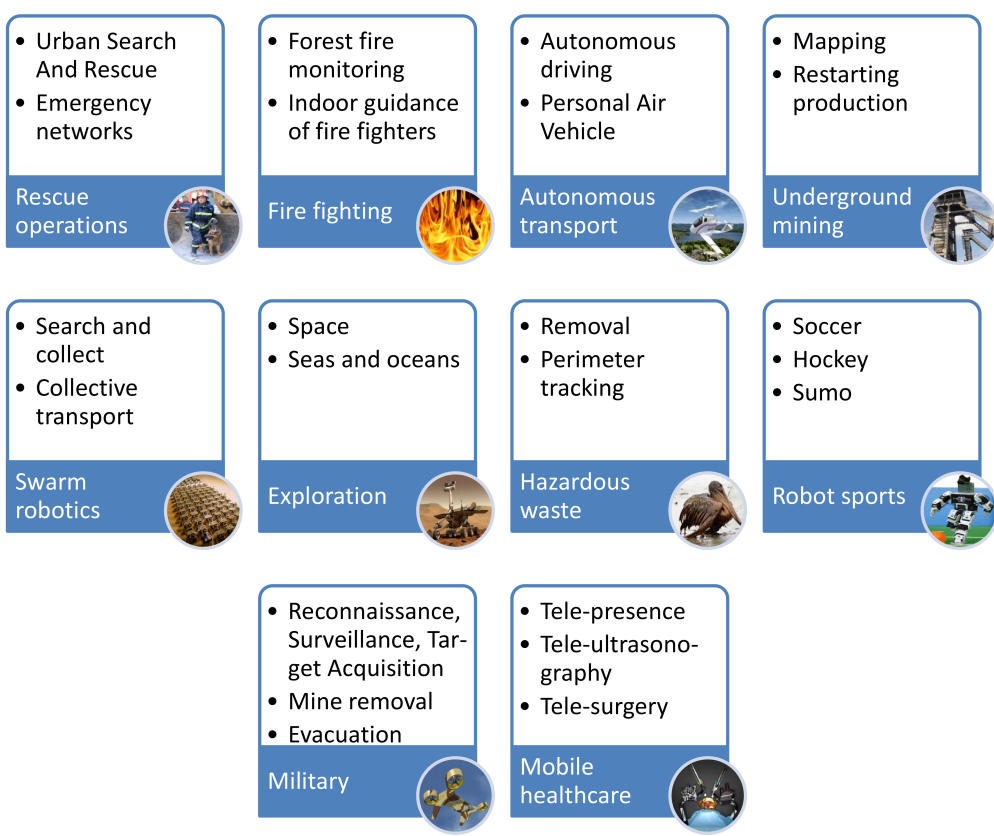

Fig. 1 Overview of uses cases where networked robots have been successfully applied

\subsection{Use cases}

\subsubsection{Rescue operations}

As illustrated in Fig. 1, networked robots have already been applied in various use cases. One of the most important cases is the utilization of networked robots in rescue operations. Several authors have presented robotic assistance during Urban Search And Rescue missions (USAR). In these cases the aim is to search for and rescue victims in disaster areas, especially those littered with debris from man-made objects such as collapsed buildings. Typically tele-operated small robots are used because they can go deeper than traditional search equipment (a camera mounted on a pole can only go 2 meters deep inside a rubble pile), they can enter void spaces too small for a human or search dog, and they can enter locations still on fire or posing great risk of structural collapse. The first known use of robots in USAR missions is the assistance of four robot teams during the rescue operations at the World Trade Center on 11 September 2001 [7]. There the robots performed well, with the primary measure being the acceptance by the rescue community. One unexpected outcome of the WTC case was the high dependency of robots on tethers (providing communication between the robot and the operator) or safety ropes (to retrieve the robot after a vertical descent or when the robot has flipped over). These techniques are characterized by a significant disadvantage: they tangle. During the rescue phase, a robot tether got tangled and could not be retrieved without an intercession. Wireless 
solutions were proven to be not mature enough, since the only wireless robot that was deployed at the WTC was lost in the field due to wireless dropout. Since then, many research efforts have focuses on improving both the mobility platform of the robots and the wireless communication between the robot and the operator. To extend the communication range to practically usable levels, a multi-hop chain network is typically proposed. In general, the intermediate hops are also mobile robots, which automatically position themselves in order to maintain the communication link between the actual rescue robot and the human operator [2,3,8-10]. A variation on this USAR approach is to rely on robots during rescue operations for the deployment of a mobile communication backbone [11]. In this case, the robots do not perform any search or rescue task, but the wireless network that they automatically deploy and maintain provides wireless communication capabilities between all rescue workers on site. Voice, video, digital maps of the area, sensor information and so on can be communicated between the different active rescue teams and the central command post, allowing more efficient management of the rescue operations.

\subsubsection{Fire fighting}

Another area where robots can be of assistance is fire fighting. Real-time monitoring of the evolution of the fire is very useful in the case of forest fire. This monitoring involves dynamic information about the fire front and other parameters such as the flame height and the fire front width. Aerial camera's are usually deployed on board of conventional aerial means such as helicopters or airplanes. Flights near the fire, with low visibility conditions due to smoke, could involve significant risk. Using Unmanned Aerial Vehicles (UAV), these risks can be avoided. The UAV approach can also decrease operational cost. Ollero et al. [12] presented such a helicopter teleoperation system that demonstrated a good overall performance during an experiment in May 2003 at the Gestosa (Portugal) forest fire. Another fire fighting use case is the search for victims in a burning industrial warehouse. This scenario is regarded as a major concern by fire fighters because of the combination of the enormous dimensions of the warehouses and the expected low visibility when smoke develops. Penders et al. [13] developed a robot swarm to support human beings searching the warehouse. In this system, the human fire fighters are fully autonomous and go their own way. The robot swarm surrounds the fire fighter in a loosely defined and flexible formation. If required, some robots leave the swarm to maintain network connectivity between the fire fighter and the command post at all times. The robots rely on their sensors for simultaneous localization and mapping (SLAM) of the burning warehouse. By combining the information produced by all members of the swarm, the fire fighter can be informed of hazards or guided towards the fire, the exit and other fire fighters.

\subsubsection{Autonomous transport}

Autonomous vehicles are also considered often as a form of mobile robots. In the paradigm of cooperative autonomous driving, a plethora of fully automated vehicles 
can cooperate in terms of cognition and trajectory planning while participating in traffic. In relatively conflict free zones such as highways this seems quite feasible, adaptive cruise control and lane keeping systems are already commercially available on several car models. However, maneuvering is still a challenging aspect of these systems. Baber et al. [14] demonstrated solutions for unsignalized intersection traversal and an overtaking maneuver by autonomous road vehicles. Nagel et al. [15] defined the communication system requirements for this application, pointing out the importance of low latency. The dramatically varying vehicle density and mobility were also identified as important aspects of this scenario. Xiong et al. [16] focused on flocking of multiple unmanned vehicles. This refers to the formation and maintenance of a desired pattern by a group of mobile vehicles without collision during movement. Milanes et al. [17] focused on the heterogeneity of future cooperative autonomous driving systems. Their experiments on a closed track demonstrated that given a well defined communication standard, different vehicles with different architectures and different control systems can cooperate using the data they exchange and a common decision algorithm to perform complex cooperative maneuvers. Another interesting type of autonomous vehicle is the Personal Air Vehicle (PAV). This is a modification of a small, personal helicopter that can also be driven on the road like a car. While in the air, the vehicles will fly by itself, under the guidance of an air traffic management system. On the ground, the PAV is driven as a standard road vehicle. Working radio-controlled models of a PAV have been built and tested by Boeing in the scope of a PAV feasibility analysis [18].

\subsubsection{Underground mining}

Underground mines are very specific environments were robots can provide an added value. Abandoned mines pose significant threats to society. Accidents have already taken place where miners in their routine work accidentally breached a nearby abandoned mine and almost drowned in the millions of gallons of water that poured upon them. Structural shifts can also cause collapse on the surface above abandoned mines. Ground water contamination is another problem of great importance. Despite of all these threats imposed on society by abandoned mines, a large fraction of them lacks accurate maps. Main cause is the fact that abandoned mines are usually not accessible to people. Lack of structural soundness is one reason, another is the harshness of the environment (low oxygen level, flooding), and the danger of explosion of methane (a gas that frequently accumulates when mines are no longer ventilated). Thrun et al. [19] developed a robotic system designed to autonomously explore and acquire three-dimensional maps of abandoned mines. Good results were obtained in field tests, but it was also noticed that the ability to communicate with a robot while inside a mine would have great operational benefits. A network of wireless repeater stations was indicated as a viable extension to mine mapping robots. A similar conclusion was drawn by Murphy et al. [20], which applied tethered robots in mine rescue and recovery operations. First steps towards such a wireless solution were taken by Weiss et al. [21], which developed a statistical model for the radio range between networked robots deployed in an underground mine. 


\subsubsection{Swarm robotics}

Wireless communication is one of the key elements of swarm robotics. This kind of systems deal with large numbers of homogeneous robots which only have rudimentary capabilities. The robots are autonomous, have limited memory and have very limited self-control capabilities. Even though each individual is only able to perform at the level of reflex actions, the local interactions of the robots may result in complex behavior at the corporate level $[22,23]$. Robot swarms are most useful for non time-critical applications involving numerous repetitions of the same activity over a relatively large area. Example use cases are searching and collecting tasks (food harvesting [24], collecting rock samples on distant planets [25]) or collective transport of palletized loads [26].

\subsubsection{Exploration}

Networked robots can also facilitate exploration of harsh environments such as space, oceans or volcanoes. One of the most famous examples are the twin Mars Exploration Rover (MER) vehicles which landed on Mars in the course of January 2004 [27]. Such rovers have the ability to move on the planetary surface, allowing them to explore different locations within the landing site. This greatly increases science return. For direct-to-Earth communications the rovers relied on relay communications through two science data gathering spacecrafts currently in orbit around mars. The MER vehicles explored Mars for more than six years. One of the most important scientific findings of this mission include incontrovertible evidence for past liquid water at both landing sites. It seems likely that in future similar projects the exploring robots will not only be tele-operated from earth, but will also form teams of semiautonomous cooperating robots. Rooker and Birk [28] developed suitable algorithms for such communicative exploration. These algorithms create a population of possible moves of every robot during every time step, weighing the benefits of exploring unknown territory versus the goal of keeping communication intact. Such exploration teams will rely on local wireless communication to coordinate their cooperative efforts, since the large physical distance between remote planets and Earth results in latency values of minutes instead of milliseconds on typical Earth-based communication systems. Toung et al. [29] successfully applied the existing ad hoc routing protocol Dynamic Source Routing (DSR) in such a scenario. Besides operations on planet surfaces, other aspects of space exploration can involve networked robots. An example is the case of autonomous spacecraft proximity maneuvers as orbital rendezvous and assembly. Bevilacqua et al. [30] researched the wireless ad hoc network technology that would be required to support such a scenario.

Robots can also be used to explore harsh environments on Earth. Much is still to be discovered of the seas and oceans, several authors have proposed autonomous undersea robot systems for this task. Wireless acoustic networking is regarded as the enabling technology for such underwater applications. However, it is affected by many challenges. The propagation delay is five orders of magnitude higher than in electro-magnetic terrestrial channels, the underwater acoustic channel suffers significantly from multipath and fading, bandwidth depends on the transmission distance 
and high bit error rates and temporary losses of connectivity can be experienced [31]. To overcome these problems, Vasilescu et al. [32] proposed a self-synchronizing time division multiple access (TDMA) scheme to schedule messages. Benton et al. [33] on the other hand proposed modifications to the Dynamic Source Routing (DSR) ad hoc networking protocol that make it suitable for undersea operation.

\subsubsection{Hazardous waste management}

Incidents with hazardous waste can be very challenging to manage. In case of chemical spills, the substance can be very harmful for humans, greatly hampering the removal of the spill. In case of oil-slicks caused by incidents with tankers or drilling rigs, management of the cleanup operation can be quite challenging because of the sheer size of the contaminated area. Robots can assist in both cases. Parker [22] developed a software architecture that facilitates the cooperative control of heterogeneous teams of mobile robots. To demonstrate the viability of this architecture, it was tested with a team of robots performing a laboratory version of hazardous waste cleanup. In this experiment, the robots are required to find the initial locations of two spills, move those spills to a goal destination, and periodically report the team's progress to a human monitoring the mission. The positive outcome of this experiment indicates the usability of networked robots for the cleanup of spills which are very harmful for humans. In the other waste management case, where contaminated areas are very large, perimeter tracking is a task that can be successfully executed by robots. In this case a group of robots is able to autonomously monitor borders that are of unknown shape and size and change in time. This real-time information can be of high importance for the management of the cleanup operations. Clark and Fierro [34] and Kingston et al. [35] focused on this use case.

\subsubsection{Robot sports}

Robot sports are very useful generic use cases for the academic research of serveral fields related to robotics. The RoboCup championships grew to be the most important robotic competition worldwide. Every year, several hundreds of teams originating from all over the world compete there. More then 70 robot competitions are organized, such as robot soccer, sumo, hockey, weightlifting, combat, stair climbing, autonomous navigation, fire fighting, etc [36]. On of the most popular competitions is the RoboCup Humanoid League, where fully autonomous soccer robots with a human-like body compete with each other. Humanoid soccer is considered as a benchmark problem for artificial intelligence research and robotics for several reasons. It requires multiple players of one team to cooperate in a dynamic environment. Sensory signals must be interpreted in real time to take appropriate actions. A soccer competition does not test isolated components, but two integrated systems compete with each other. The number of goals scored is an objective performance measure that allows comparison of systems that implement a large variety of approaches to perception, behavior control and robot construction. The presence of opponent teams, which continuously improve their system, makes the problem harder every year. Such a challenge focuses the effort of many research groups worldwide 
and facilitates the exchange of ideas. [37]. Technological advancements originating from robot soccer can be applied later in many other use cases. An example is the study of Weitzenfeld [38] which extended robotic architectures originally developed for RoboCup with additional capabilities to perform surveillance missions.

Besides an accelerator for academic research, robot sports have also proven to be an interesting teaching instrument. A nice example is the work of Fiene [39] that describes an approach to mechatronics education based on robot hockey. Each year, groups of engineering students are substantially trained in mechanical design, electronics and programming during their quest to create the best university team of three autonomously hockey-playing robots.

\subsubsection{Military}

One of the more familiar applications of wireless robots are the military use cases. Unmanned Aerial Vehicles (UAV) such as the Predator series of the US army are quite known by the general public because of news coverage of their operations in Afghanistan and Iraq. These operations fall within the type of use cases called Reconnaissance, Surveillance and Target Acquisition (RSTA). The Predator UAVs are tele-operated through satellite communication systems. Future systems will likely consists of teams of UAVs that execute missions in a (semi-)autonomous manner. This requires the development of distributed UAV decision and control systems that comprise three layers: flocking, communication and task assignment. Ben-Asher et al. [40] have researched these different aspects. Other trends are the deployment of Unmanned Ground Vehicles (UGV) during RSTA missions. Luu et al. [41] presented a system based on mobile ad hoc networking that enables a soldier to remotely control a UGV. It was tested in both rural and urban outdoor environments. Nguyen et al. [42] also focused on the UGV case, but with an emphasis on indoor usage. They developed a system where a lead robot is assisted by mobile relay robots which follow the lead robot and automatically stop where needed to maintain a solid communication network between the lead robot and the operator. Relay nodes that become unnecessary because of a changed position of the lead robot catch up with that lead for later reuse. Hsieh et al. [43] presented a multi-robot team consisting of two UAVs and eight UGVs which cooperate during urban surveillance missions. The team is able to monitor a small village, and search for and localize human targets by the color of the uniform, while ensuring that the information from the team is available to a remotely located human operator.

Besides in RSTA missions, networked robots are researched and applied by military organizations in a much broader spectrum of use cases. Mine removal is a topic that received quite some attention. Naval mine countermeasure operations are taken to counter the effectiveness of underwater mines. These missions include finding and seizing mine stockpiles before they are deployed, sweeping desired operational areas, identifying mined areas to be avoided, and locating and neutralizing individual mines. The research of Sariel et al. [44] focused on the subset of such operations that involve locating and mapping all individual mines in an operational area. Their experiments showed that communication delays significantly influence the solution quality and should be analyzed in multi-robot systems. Lindemuth et al. [45] focused 
on mine countermeasure operations in shallow water. The presented system consists of a floating unmanned surface vehicle that has the capability to deploy an UAV when needed. Together these two robots provide all required sensors under and above the waterline to support any mine countermeasure operation. On land, quite different challenges are to be faced when disarming mines. Pezeshkian et al. [46] focused on explosive ordnance disposal operators using robots to defeat improvised explosive devices. Their research is an evolution of the work of Nguyen that focused on indoor RSTA missions with mobile relay nodes. In this evolution, the mobile relays are replaced by static relay nodes that are carried and dropped by the tele-operated robot to maintain its communication link. Such static nodes are more practical and costefficient than their mobile counterparts, and can be retrieved after completion of the mission for future reuse. Effective communication range between operator and robot, a key characteristic during explosive disposal missions, could be greatly increased with this approach.

A third military use case is rescuing the wounded in the battlefield. Nguyen et al. [47] developed a robotic medical patient transport system where the track of a human leader, with a GPS unit in his backpack, was sent wirelessly to the robotic transporter, also equipped with a GPS unit. The transporter traced the path of the human leader by following these GPS breadcrumbs. Without the robotic assistance, two to four persons are required per wounded, but with this system one person can transport several wounded. This saves care givers valuable time, allowing them to focus on providing immediate medical treatment instead of the more logistical task of transportation. Jiang et al. [48] omitted the concept of a human leader, avoiding human intervention during rescue operations in the extremely dangerous battlefield. Such a system is characterized by a higher level of complexity: all mobile robots have to be notified about entities waiting for rescue, rescue tasks have to be allocated among the mobile robots, and mobile robots have to be guided to reach the designated targets without location information. Three different schemes for fulfilling these requirements were investigated.

\subsubsection{Mobile healthcare}

Mobile healthcare (m-health) has been defined as "mobile computing, medical sensor, and communications technologies for healthcare". This concept represents the evolution of a e-health system from traditional desktop tele-medicine platform to wireless and mobile configurations [49]. Within this domain, several use case are found in literature which rely on networked robots. Petelin et al. [51] described their experiences with a tele-presence robotic system that was field tested in a hospital for approximately 4 months. This system is composed of a control station at a central location, a mechanical robot, a wireless network at the hospital and a high-speed Internet connection at both the hospital and the central location. It allows the central party (e.g. physician) to control the movements of the robot itself, see and hear at the remote location (hospital), and be seen and heard at the remote location (hospital) while not being physically there. The patient and nursing personnel response has been very positive during the test. The main two advantages were significant time 
profits in the discharge process (usually 4 or more hours earlier than previously) and increased access to physicians during "off-hours".

Garawi et al. [49] developed a system for wireless robotic tele-ultrasonography. It comprises a fully portable tele-operated robot allowing a specialist sonographer to perform a real-time robotized tele-echography (ultrasonography) to remote patients. The advantage is that small medical centers can rely on specialized expertise available in other centers when specific unusual ultrasonography examinations have to be performed. Because the robot is equipped with $3 \mathrm{G}$ communication capabilities, it can also be installed in rescue vehicles, as demonstrated later by Takeuchi et al. [50]. This allows the remote examination of the patient during the transportation to the hospital, saving valuable time in case of emergency.

Advances in robotics and computing have resulted in surgical robots that are currently used in operating rooms across the world. The next step is the evolution from robotic surgery to robotic tele-surgery. In this case high bandwidth terrestrial communications are used to allow a remote surgeon to operate on a patient regardless of the patient's location or environment. Harnett et al. [52] focused on the use case of tele-surgery in an extreme environment where robust broadband communication networks are not available: the battlefield. They presented a surgical robot that relies on an intermediate networked UAV to provide the required communication link between surgeon and robot. In their experiments they demonstrated that surgeons were able to remotely perform several simple surgical tasks such as suturing in the extreme battlefield environment.

\subsection{Robotic Ad Hoc Networks}

As indicated by Wang et al. [53] and illustrated in Fig. 2, an evolution in the domain of robot wireless communication can be clearly distinguished over the last decades. In the early phases, infrared technology was applied on a large scale because of its low cost. The main goal was the provision of capabilities for analog tele-operation of the wireless robot. But infrared waves cannot pass through obstacles and are influenced by light and weather circumstances. Data rate of this technology is also limited. Therefore radio frequency (RF) technology became more preferred in the design of mobile robot communication. First RF technology was primarily used for point-to-point links, still with a focus on analog tele-operation of the wireless robot. Most solutions operated in the Industrial Scientific Medical (ISM) band, which is a license-free frequency band at $2.4 \mathrm{GHz}$. In a later phase, researchers started exploring the capabilities of using proliferating Internet-like wireless technologies such as IEEE 802.11 Wireless LAN and the Bluetooth standard. These technologies also operate in the ISM band, but were originally designed for the communication of digital data at high speeds. This resulted in a transition from analog tele-operation of the robot to the extensive collection of use cases for networked robots that was discussed in section 2.1. On of the biggest challenges in realizing these applications is the short communication range of the IEEE 802.11 and Bluetooth technologies. Therefore most authors rely on Robotic Ad Hoc Networks (RANET) to provide the required robot wireless 


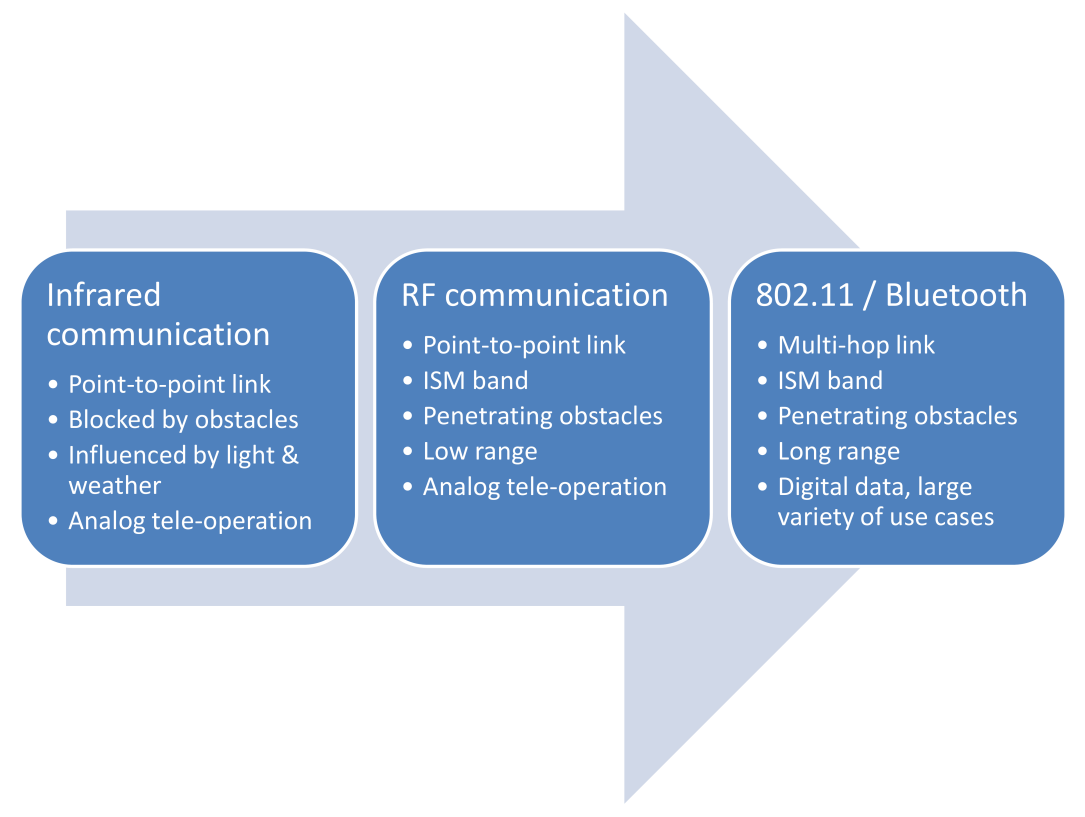

Fig. 2 Evolution of robot wireless communication over the last decades

communication capabilities. In fact, except for the domain of mobile healthcare, all use cases described in section 2.1 utilized RANET networking solutions.

As explained in section 1, a RANET protocol enables the robots to form a wireless ad hoc network where there are no fixed routers, all nodes are capable of movement and can be connected dynamically in an arbitrary manner. Nodes of these networks function as routers which discover and maintain routes to other nodes in the network. The design of RANET protocol encompasses two quite different dimensions: wireless ad hoc networking protocols, and movement control protocols. The former describes how information has to be forwarded by intermediate nodes in a connected ad hoc network, the latter describes how mobile robots can influence their own movement to guarantee that the network is connected. The related work regarding the ad hoc networking aspect of RANETs can be further categorized based on three different design approaches. Several authors experimented with existing MANET protocols in the context of networked robots. Their main research goal is to assess the suitability of these protocols for the RANET use case. The only considered optimization is a suitable tuning of the protocol parameters. A second group of authors investigated if these existing MANET protocols could be adapted or extended to better support RANET networking. A third group of authors preferred a clean slate approach, designing a specific RANET ad hoc networking protocol from scratch. In the next subsections, the related work regarding these different aspects of robotic ad hoc networking is introduced. An overview of this survey is given in Fig. 3. 


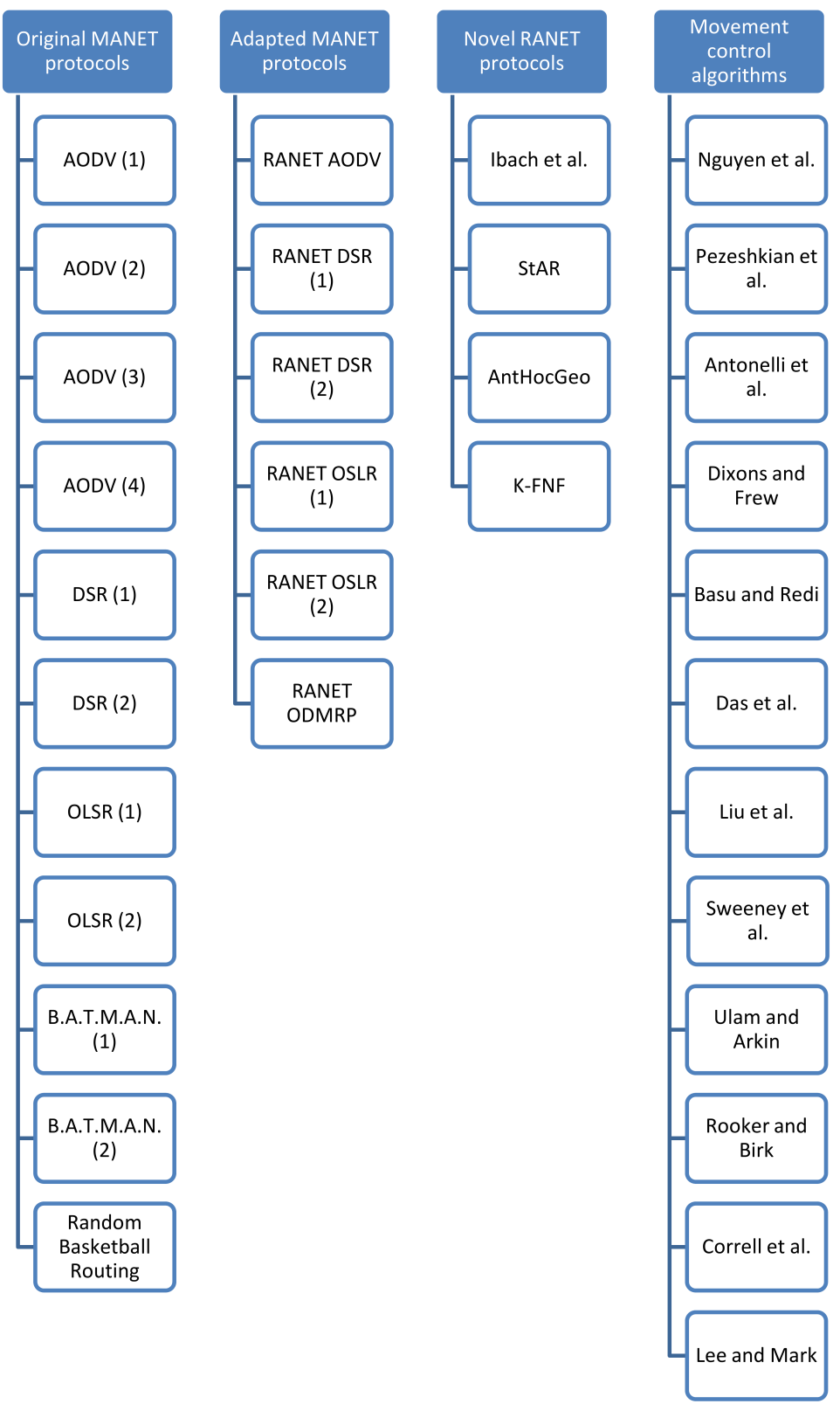

Fig. 3 Overview of the related work in the RANET domain. For every aspect of RANET networking, it is indicated which techniques were proposed in literature. For every technique, the amount of studies that focused on that technique is also given. If techniques where found without a name, a reference to the authors was given. If multiple nameless techniques would have shown strong resemblance, they would have been given a collective name to illustrate the similarities. This was however never required. 


\subsubsection{Original MANET protocols}

When investigating the capabilities of unchanged existing MANET protocols, several authors focused on the Ad hoc On-demand Distance Vector (AODV) protocol. This is a reactive routing protocol since it only establishes a route between a sourcedestination pair when the source node has data to send to the destination. The route establishment phase of the protocol consists of two main control messages, the route request (RREQ) and the route reply (RREP). The RREQ is sent by the source node, and flooded over the network until it reaches the destination. During that process the intermediate nodes update their routing tables to establish a reverse path to the source. The destination responds with a RREP which is unicasted back towards the source to establish the route. For route maintenance, hello messages are utilized. For more details we refer to RFC 3561 [54]. Pohjola et al. [55] applied AODV in a scenario where the actions of the robots within a squad of 25 robots are controlled by a single squad leader. The size of the formation compared to the limited radio range of 15 meters ensured that multi-hop communication was required. The speed of the robots was constrained to approximately $6 \mathrm{~km} / \mathrm{h}$. For such a limited mobility, AODV performed in an acceptable manner, because of the strong resemblance of a standard scenario for mobile ad hoc networking. However, in most robotic use cases, the speed of movement will be significantly higher, and robots will not always tend to stay together in a squad. In this case, AODV is an inadequate protocol, as demonstrated by Weitzenfeld et al. [38], Kudelski et al. [56] and Zeiger et al. [57].

Similar results were obtained with the Dynamic Source Routing (DSR) protocol. This is another reactive ad hoc routing protocol, which works similar to AODV. One of the most important differences is the fact that during the route establishment phase, the reverse route is not defined by the routing tables of the intermediate hops, but by route information that is added to the route request packet by the intermediate hops. Route maintenance is also based on acknowledgment packets instead of hello messages [58]. Toung et al. [29] achieved positive results with the DSR protocol in a scenario for planetary exploration. However, the nodes in the network did not move at all in the described experiments. Zeiger et al. [57] tested DSR in a scenario where the movement of one mobile robot (3-5 $\mathrm{km} / \mathrm{h}$ ) forced the protocol to increase the number of participating nodes in the communication link. Although DSR performed slightly better than the other MANET protocols tested in that publication, it still suffers from a packet error rate of more then $11 \%$ at these very low movement speeds. The other tested protocols in the study where AODV, OLSR (Optimized Link State Routing) and B.A.T.M.A.N. (Better Approach To Mobile Ad hoc Networking). OLSR is a proactive table-driven ad hoc networking protocol. It continuously maintains routes to all destinations in the network, and distributes link and neighborhood information between nodes in each others neighborhood using hello messages. These are also used for route maintenance. To minimize the overhead of flooding, OSLR uses only selected nodes (multipoint relays) to retransmit control messages [59]. B.A.T.M.A.N. is a new approach to ad hoc networking, this protocol does not calculate routes. It continuously assesses its neighborhood by receiving and broadcasting packets from other nodes. Instead of discovering the complete route to a destination node, the protocol only identifies the best single-hop neighbor and forwards the packet to this neighbor. 
The time-to-live value is essential to avoid forwarding storms [57]. Both protocols performed unsatisfactory in these experiments of Zeiger et al. In later work, the same authors attempted to optimize the performance of the four tested protocols through parameter tuning [60]. For all protocols performance could be somewhat improved, but they still remained unfeasible solutions for robotic ad hoc networking.

The Random Basketball Routing protocol is a more recent MANET routing algorithm that was optimized for node mobility. Jung et al. [1] applied this protocol in their study of cooperative path finding. In this use case a team of robots cooperates in searching the exit of a random maze. Random Basketball Routing is characterized by a key parameter $\mathrm{p}$, the relay (receiving) probability. For a given time slot, a source node having data is either in transmission mode with probability 1-p or in receiving mode with probability $\mathrm{p}$. When a source node is in transmission mode, it sends its data to either a relay node or the destination node if the destination is within the one-hop communication range. When a source node is in receiving mode, it listens to the other transmitting nodes for relay. A source node is responsible for the selection of the next relay node. This is based on polling of the candidate relay nodes within radio range, and reporting of the measured signal strength of received periodic beacons. In this study, the experiments focused on the performance of the algorithm for cooperative path finding, and not on the supporting routing protocol. The executed experiments consisted of 3 mobile robots traveling at speeds below $1 \mathrm{~km} / \mathrm{h}$. The only information regarding the network performance is the fact that the cooperative path finding algorithm could be successfully applied. Hence no conclusions can be drawn regarding the feasibility of applying Random Basketball Routing in a wider variety of networked robots use cases.

\subsubsection{Adapted MANET protocols}

Instead of parameter tuning, several authors focused on the adaption or extension of existing MANET protocols. A profound study was performed by Das et al. [61]. In this work, adapted RANET versions of the AODV, DSR and ODMRP protocols were presented. To optimize AODV, nodes apply probabilistic rebroadcasting of the RREQ messages. The corresponding probability value is defined in such a way that highly mobile robots are not likely to rebroadcast a RREQ message. This increases route lifetime and reduces routing overhead and energy consumption. Every hop that decides to rebroadcast the RREQ will also calculate a timeout value that indicates when the robot will move out of the range of the route being set up. This information is injected in the RREQ packet, and distributed to all nodes on the route in the RREP message. It will then be used by the protocol to efficiently manage and utilize routes before they break. The same techniques (probabilistic forwarding of RREQ and usage of timeout value for route management) were also introduced in the DSR protocol and in the ODMRP (On Demand Multicast Routing Protocol) protocol. ODMRP is multicast protocol developed for MANETs which uses a mesh to enable redundancy and consequently more reliable delivery. The performance evaluation of the three adjusted protocols demonstrated a significant increase in control messages overhead and power consumption in all cases. However, packet delivery rate and average delay were not improved. Benton et al. [33] also focused on the DSR protocol, 
they developed a simplified DSR implementation tailored to the unique needs of the undersea network environment. The optimizations focused on smaller groups operating in a low bandwidth environment. The obtained results were promising, but only applicable for this very specific use case.

Tiderko et al. [62] presented a communication framework for a multi-robot system that relies on an adapted version of the OSLR protocol. They extended the hello and topology control management frames with additional information about link attributes. These attributes (received signal strength, loss rate of a link and the utilization of the air interface) are used to estimate the link quality. This link quality value influences the outcome of the Dijkstra algorithm that calculates the route for a given source-destination pair. The performance analysis of this solution was however too limited to assess the suitability of the proposed solution as a solid RANET protocol. Milanes et al. [17] also adjusted the OLSR protocol. Their focus was to limit the area in which topology information is relayed and to rapidly build small ad hoc networks that are well suited to the dynamic context of autonomous vehicle communications at a crossing. The intended use case could be supported with the proposed technique, but because of its focus on small topologies it cannot be considered as a suitable generic RANET protocol.

\subsubsection{Novel RANET protocols}

A few authors presented novel protocols that were specifically designed from the ground up to support RANET communications. Ibach et al. [23] proposed an algorithm that employs clustering of mobile nodes in order to minimize the number of control messages that flood into the network. This algorithm is combined with techniques for position based routing. However, no performance measurements were executed. Hence it can not be evaluated if this proposed protocol performs satisfactory.

Weitzenfeld et al. [38] developed the StAR (Steward Assisted Routing) protocol for networks in which links are often unavailable due to mobility or other interference. The objective of the protocol is to nominate, for each partition in the network, a steward for each destination. These stewards are the robots that are next expected to have communication with the destination. For example, if there is a single moving robot who communicates with all other stationary nodes, this robot is likely to be nominated as the steward for all destinations. Messages are sent to the associated steward, which will store them until a route to the destination (or better steward) is available. StAR routes messages using a combination of global (network-wide) contact information and local (intra-partition) route maintenance. Several small-scale experiments with the protocol where presented. The results demonstrated the capabilities of the protocol to successfully handle scenarios with unreliable links or movement of a part of the network nodes. However, no information was given regarding the speed of the movement, a scenario where all nodes are mobile is missing, and no conclusions can be derived regarding the ability of the protocol to handle large-scale scenarios.

The AntHocGeo protocol was proposed by Kudelski et al [56]. This routing scheme uses geographical cells to comprise the routing information within the geographical location. This means that a path between a given source and destination is 
defined as a list of consecutive geographic locations instead of consecutive network nodes. As a result, the protocol is less influenced by the movement of individual nodes in the RANET, since the path can be considered alive as long as there exists a mobile host in each of its cells. Besides geographical cells, the AntHocGeo protocol also relies on elements from an ant routing algorithm based on the ant colony optimization metaheuristic. The protocol was tested in indoor scenarios for mobile robot networks. The number of nodes was varied between 5 and 50, the speed of the nodes between 4 and $18 \mathrm{~km} / \mathrm{h}$. The indoor environment consisted of 13 connected rooms. For these scenarios, the protocol performed well. This protocol seems promising, but further research is needed to confirm the protocol's capability of handling scenarios with much faster node movement.

Jiang et al. [48] presented the k-FNF scheme. When a specific node detects the occurrence of an event, it broadcasts an event notification packet throughout the entire network. To eliminate the redundant forwarding packets typical for standard flooding schemes, the k-FNF scheme utilizes received signal strength to determine the backoff time for rebroadcast of the packet. Farther nodes get weaker signals and have shorter backoff times. Besides, a node does not forward the packet if it hears k forwarded packets before the backoff time elapses. This can reduce a lot of redundant forwarding packets, saving energy and resulting in better network scalability characteristics. The performance evaluation confirmed the capability of the protocol to support large-scale networks. However, all nodes in the network were static, no mobility aspect was taken into account. Another downside is that fact that the protocol only supports message broadcasting, unicast or multicast traffic is not supported. Hence it may be considered as an interesting part of a future RANET solution, but not as global RANET protocol.

\subsubsection{Movement control algorithms}

Mobile networked robots can influence their own movement to guarantee that the network is connected. This is a unique characteristic of RANET networking. Several authors already presented appropriate movement control algorithms. Nguyen et al. [42] proposed a system where mobile autonomous communication relay nodes convoy behind a lead robot and automatically stop when needed to maintain a solid communication network between the lead robot and a remote operator. In tactical and reconnaissance missions, the robot's convoluted path may often lead to situations where intermediate relay nodes are no longer needed. To maximize resources and allow for extended explorations, unneeded relay nodes catch up with the lead robot to be redeployed later. This research of Nguyen et al. was continued in the work of Pezeshkian et al. [46]. The use case shifted from reconnaissance missions to tactical missions such as explosive ordnance disposal. As a result, the use of mobile relays nodes was no longer required. Instead, it was chosen to equip the lead robot with a deployment system capable of carrying relay-radios that are automatically released when needed in order to maintain the communication link back to the control station. Once dropped off, these so called Relay Bricks can turn itself upright and raise its antenna to establish proper radio connectivity. This approach is more practical and more cost efficient than in the case of mobile relay nodes. As demonstrated in both outdoor as mixed indoor/outdoor experiments, the presented system introduced a significant 
increase in the maximum supported distance between the tele-operated robot and the human operator. In later work [63], several hardware optimizations were presented, but the main principles remained unchanged.

Similar work as that of Nguyen et al. was performed by Antonelli et al. [9], which also focused on the scenario where a platoon of mobile relay robots is applied to guarantee a constant communication between a rescue robot and a fixed base station. In this study, the control objective is achieved in the framework of a kind of behavioral control, namely the Null-Space-based Behavioral control. This decomposes the overall mission of the system in elementary tasks, solves them as they were working alone, and finally combines the outputs of the single tasks to obtain the motion command for each robot. The tasks that each relay node has to satisfy are: obstacle avoidance, keeping the next relay in the comfort zone, keeping the previous relay in the comfort zone, and reaching a target. The simulation analysis of this solution illustrated some preliminary encouraging results. Another publication that focused on the same scenario is the work of Dixon and Frew [64]. Their decentralized mobility control algorithm relies on measurements of the signal-to-noise values for the links between a relay node and its two communication neighbors, and not on geographic position or communication range. This allows a communication chain of robotic relays to respond to changes and unexpected features in the RF environment, which is not possible with position based chaining solutions. Simulations showed that this algorithm will drive a team of robotic vehicles to locations that achieve the global objective of maximizing capacity of a cascaded communication chain, even in the presence of an active jamming source.

Basu and Redi [65] focused on fault tolerance. Starting from an arbitrary initial connected configuration, the goal was to move a subset of robot nodes from their initial locations to a new set of locations such that the new connectivity graph is more tolerant to node failures than the initial graph. Two heuristic algorithms to achieve this goal were investigated. The first algorithm is called contraction. In this case all nodes include their location information in the link state updates that are flooded over the network. Using this information, every node in the network can calculate the geographic center of the entire network. Then every node independently moves towards this center by a weighted distance determined by a parameter called the contraction parameter. The second algorithm is called block movement. It focuses on the identification and removal of cutvertices in the network graph. A cutvertex is a vertex that breaks a connected graph into two or more connected components if it would be removed. Biconnected components are called blocks. The algorithm tries to move blocks in such a way that the global graph remains connected but that no cutvertices are present any more. The authors concluded that the iterative block movement algorithm significantly outperforms the contraction heuristic in the total distance traveled metric. The work of Das et al. [66] focused on the same problem of turning a connected network into a biconnected network by controlling the robot movement. In contradiction to the global, centralized system of Basu and Redi, this study applied a localized movement control algorithm. In this algorithm, every nodes checks for itself if it is part of a cutvertex in a smaller sub-graph of the network that contains this node. If it indeed is a critical node, it broadcasts a critical announcement message to all its direct neighbors. Using this information, non-critical nodes can define 
their optimal movement, with a common goal of transforming all critical nodes into non-critical nodes. The performance analysis of the algorithm demonstrated that it significantly outperforms the algorithm of Basu and Redi in terms of distance traveled. Liu et al. [67] also focused on the problem of creating biconnected network graphs. They propose a distributed movement control algorithm that emulates the attractive force (such as the force in a stretched spring) and the repulsive force (such as the electrostatic force between electric charges) in nature. The attractive force pushes the node towards the center of the network, making it connected/biconnected. The repulsive force pulls the nodes away from each other, maximizing the coverage of the network. Each robot follows the resultant virtual force to move. The proposed solution can self-organize an initial and possibly disconnected network into a biconnected network, and it can maximize the coverage of the network while the total moving distance in the movement process is minimized. Good performance results were demonstrated both through theoretical proof and simulator analysis.

Sweeney et al. [8] presented mechanisms that allow cooperation without interference of movement controllers addressing multiple goals such as network maintenance, search and rescue tasks, etc. They also presented the QoS Hand Off Protocol (QHOP) for handling route discovery and repair. It takes advantage of the available information regarding the mobility of the nodes to predict routing faults before any connection is broken. Therefore, the team can actively plan to reroute traffic so that there is no loss of service. For example, if signal strength decreases, it may be due to temporary signal fluctuations, or because the sender and receiver are moving apart. Because the desired motion of the robots can be examined, it can be determined whether the signal attenuation is expected to continue, and initiate route discovery if necessary. The proposed mechanisms were evaluated in a simulation platform that combines the network simulator NS-2 with the mobile robot simulator Player/Stage. In a scenario with four robots in a $50 \mathrm{~m}$ x 50 environment, satisfactory results were achieved.

The work of Ulam and Arkin [68] addressed reactive aspects of communication recovery. It defines how members of the team should react in the event of unseen communication failures between some or all of the nodes in the network. A suite of four primitive communication recovery behaviors was formulated. The probe behavior creates an attraction to nearby open space, the retrotraverse behavior creates an attraction to waypoints stored at given distance intervals during mission execution, the move-to-higher-ground behavior creates an attraction to nearby inclines and the move-to-nearest-neighbor behavior creates an attraction to the last known position of the nearest teammate. During simulator experiments move-to-nearest-neighbor behavior proved most successful in terms of mission completion. It also provided the most rapid recovery of communication. The area covered by the robot team during surveillance missions was at its greatest when the probe behavior was applied. From these results, it was concluded that more sophisticated techniques are required that choose between the different behaviors in a situation-dependent manner. To this end, the concept of a behavior sequencer was explored.

Rooker and Birk [28] extended the concept of frontier based exploration. They presented a communicative exploration algorithm based on a utility function, which weights the benefits of exploring unknown territory versus the goal of keeping com- 
munication intact. The algorithm creates a population of possible moves of every robot during every time step. For every member of the population a utility is calculated and the one with the highest utility value is selected. For the calculation of this utility, the different options where a robot can move to have to be considered. A utility function is presented that assesses if a given move would result in a impossible position, a loss of communication or a frontier cell. Extra behavior was also presented to allow deadlock recovery. This behavior is based on meeting point strategies. The experimental results demonstrated the feasibility of the presented techniques.

The research of Correll et al. [11] focused on the deployment and maintenance by mobile robots of a wireless communication backbone. Such a network can provide communication support for disaster or military operations. The presented algorithm has minimalist requirements on the individual robotic node, namely knowledge of the number of wireless links and bumper sensors for collision avoidance. It does not require any localization. In the system, robots keep exploring the configuration space by random walk and stop only if their current location satisfies user-specified constraints on connectivity (number of neighbors). Encouraging results where obtained with the algorithm in both simulations and real life experiments. A similar scenario was investigated by Lee and Mark [69]. In this case aerial robotic vehicles were applied instead of the ground vehicles used by Correll et al. A decentralized, cooperative control strategy was presented for these aerial robot vehicles to establish a wireless communication backbone for wide area surveillance, rescue and tracking applications. The strategy consists of two consecutive phases. First optimal deployment of the aerial vehicles for high bandwidth communication networks is accomplished by applying an adaptive hill-climbing type control algorithm. With this algorithm, each aerial vehicle seeks out its own local extremum location by using the information received from neighboring aerial vehicles and remote nodes. In the second phase, when all robots have found their optimal/suboptimal location, it is necessary for the aerial vehicles to fly in a formation that minimize the effects of each robot's bank angle to maximize the communication signal strength between the aerial vehicles.

\subsection{RANET requirements}

In the previous sections a thorough survey of the domain of networked robots was given. Possible use cases, the evolution from infrared point-to-point links to the RANET paradigm and the different technical aspects of RANET networking were elaborated upon. Based on this experience and knowledge, it is now possible to define an exhaustive list of RANET requirements. In Table 1, the relationship between these requirements and the use cases described in section 2.1 is illustrated.

Some of these requirements are rather straightforward. A robotic ad hoc network should be able to operate without any fixed infrastructure. This means that when such a network is required, it should be possible to deploy it in a short term using only networked robots and optionally battery-powered stationary network relays. To ease this deployment, the network should be self-organizing: when the wireless nodes are put in place, the RANET protocol should allow these nodes to automatically find each other and configure all routing tables correctly to ensure the required multi-hop 


\begin{tabular}{|c|c|c|c|c|c|c|c|c|c|c|c|c|c|c|c|c|c|c|}
\hline & 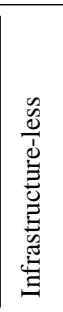 & 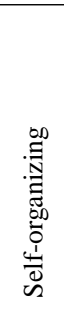 & 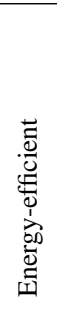 & $\begin{array}{l}\frac{0}{0} \\
\frac{\pi}{\pi} \\
\text { J } \\
\tilde{n}\end{array}$ & 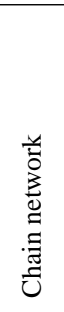 & 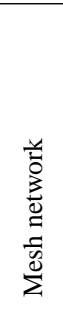 & 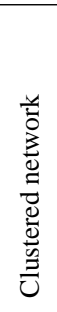 & 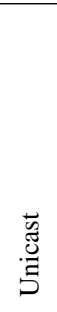 & 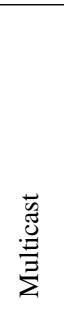 & 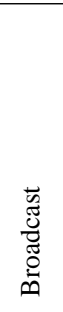 & 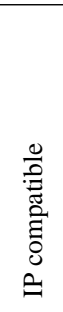 & 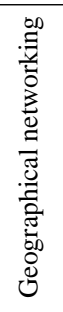 & 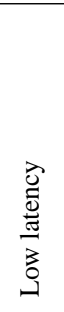 & 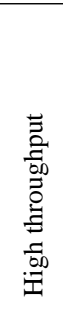 & 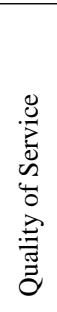 & 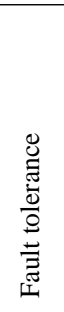 & 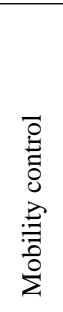 & 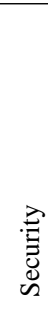 \\
\hline Urban Search And Rescue & $\checkmark$ & $\checkmark$ & $\checkmark$ & & $\checkmark$ & & $\checkmark$ & $\checkmark$ & $\checkmark$ & & $\checkmark$ & & $\checkmark$ & $\checkmark$ & $\checkmark$ & $\checkmark$ & $\checkmark$ & $\checkmark$ \\
\hline Emergency networks & $\checkmark$ & $\checkmark$ & $\checkmark$ & & & $\checkmark$ & $\checkmark$ & $\checkmark$ & $\checkmark$ & $\checkmark$ & $\checkmark$ & & $\checkmark$ & $\checkmark$ & $\checkmark$ & $\checkmark$ & $\checkmark$ & $\checkmark$ \\
\hline Forest fire monitoring & $\checkmark$ & $\checkmark$ & $\checkmark$ & & & $\checkmark$ & $\checkmark$ & $\checkmark$ & & $\checkmark$ & $\checkmark$ & $\checkmark$ & $\checkmark$ & $\checkmark$ & $\checkmark$ & $\checkmark$ & $\checkmark$ & $\checkmark$ \\
\hline Indoor guidance fire fighers & $\checkmark$ & $\checkmark$ & $\checkmark$ & $\checkmark$ & & $\checkmark$ & $\checkmark$ & $\checkmark$ & $\checkmark$ & & $\checkmark$ & & $\checkmark$ & $\checkmark$ & $\checkmark$ & $\checkmark$ & $\checkmark$ & $\checkmark$ \\
\hline Autonomous driving & $\checkmark$ & $\checkmark$ & & $\checkmark$ & & $\checkmark$ & & $\checkmark$ & & $\checkmark$ & $\checkmark$ & $\checkmark$ & $\checkmark$ & & $\checkmark$ & $\checkmark$ & & $\checkmark$ \\
\hline Personal Air Vehicle & $\checkmark$ & $\checkmark$ & & $\checkmark$ & & $\checkmark$ & & $\checkmark$ & & $\checkmark$ & $\checkmark$ & $\checkmark$ & $\checkmark$ & & $\checkmark$ & $\checkmark$ & & $\checkmark$ \\
\hline Mine mapping & $\checkmark$ & $\checkmark$ & $\checkmark$ & & $\checkmark$ & & & $\checkmark$ & & & $\checkmark$ & & $\checkmark$ & $\checkmark$ & $\checkmark$ & $\checkmark$ & $\checkmark$ & $\checkmark$ \\
\hline Restarting mine production & $\checkmark$ & $\checkmark$ & $\checkmark$ & & $\checkmark$ & & & $\checkmark$ & & & $\checkmark$ & & $\checkmark$ & $\checkmark$ & $\checkmark$ & $\checkmark$ & $\checkmark$ & $\checkmark$ \\
\hline Search and collect & $\checkmark$ & $\checkmark$ & $\checkmark$ & $\checkmark$ & & $\checkmark$ & & & $\checkmark$ & $\checkmark$ & $\checkmark$ & $\checkmark$ & $\checkmark$ & & & & $\checkmark$ & $\checkmark$ \\
\hline Collective transport & $\checkmark$ & $\checkmark$ & $\checkmark$ & $\checkmark$ & & $\checkmark$ & & & $\checkmark$ & $\checkmark$ & $\checkmark$ & $\checkmark$ & $\checkmark$ & & & & $\checkmark$ & $\checkmark$ \\
\hline Space exploration & $\checkmark$ & $\checkmark$ & $\checkmark$ & & & $\checkmark$ & & $\checkmark$ & & & $\checkmark$ & & $\checkmark$ & & $\checkmark$ & $\checkmark$ & $\checkmark$ & $\checkmark$ \\
\hline Underwater exploration & $\checkmark$ & $\checkmark$ & $\checkmark$ & & $\checkmark$ & & & $\checkmark$ & & & $\checkmark$ & & $\checkmark$ & $\checkmark$ & $\checkmark$ & $\checkmark$ & $\checkmark$ & $\checkmark$ \\
\hline Waste removal & $\checkmark$ & $\checkmark$ & $\checkmark$ & & $\checkmark$ & & & $\checkmark$ & & & $\checkmark$ & & $\checkmark$ & $\checkmark$ & $\checkmark$ & $\checkmark$ & $\checkmark$ & $\checkmark$ \\
\hline Waste perimeter tracking & $\checkmark$ & $\checkmark$ & $\checkmark$ & & $\checkmark$ & & & $\checkmark$ & & & $\checkmark$ & & $\checkmark$ & $\checkmark$ & $\checkmark$ & $\checkmark$ & $\checkmark$ & $\checkmark$ \\
\hline Robot soccer & $\checkmark$ & $\checkmark$ & $\checkmark$ & & & $\checkmark$ & $\checkmark$ & $\checkmark$ & $\checkmark$ & $\checkmark$ & $\checkmark$ & $\checkmark$ & & $\checkmark$ & $\checkmark$ & & & \\
\hline Robot hockey & $\checkmark$ & $\checkmark$ & $\checkmark$ & & & $\checkmark$ & $\checkmark$ & $\checkmark$ & $\checkmark$ & $\checkmark$ & $\checkmark$ & $\checkmark$ & & $\checkmark$ & $\checkmark$ & & & \\
\hline Robot sumo & $\checkmark$ & $\checkmark$ & $\checkmark$ & & $\checkmark$ & & & $\checkmark$ & & & $\checkmark$ & & $\checkmark$ & & & & & \\
\hline RSTA & $\checkmark$ & $\checkmark$ & $\checkmark$ & $\checkmark$ & $\checkmark$ & $\checkmark$ & $\checkmark$ & $\checkmark$ & $\checkmark$ & $\checkmark$ & $\checkmark$ & $\checkmark$ & $\checkmark$ & $\checkmark$ & $\checkmark$ & $\checkmark$ & $\checkmark$ & $\checkmark$ \\
\hline Mine removal & $\checkmark$ & $\checkmark$ & $\checkmark$ & & $\checkmark$ & & & $\checkmark$ & & & $\checkmark$ & & $\checkmark$ & $\checkmark$ & $\checkmark$ & $\checkmark$ & $\checkmark$ & $\checkmark$ \\
\hline Evacuation & $\checkmark$ & $\checkmark$ & $\checkmark$ & & $\checkmark$ & & & $\checkmark$ & & & $\checkmark$ & & $\checkmark$ & $\checkmark$ & $\checkmark$ & $\checkmark$ & $\checkmark$ & $\checkmark$ \\
\hline Tele-presence & & $\checkmark$ & $\checkmark$ & & $\checkmark$ & & & $\checkmark$ & & & $\checkmark$ & & $\checkmark$ & $\checkmark$ & $\checkmark$ & & & $\checkmark$ \\
\hline Tele-ultrasound & & $\checkmark$ & $\checkmark$ & & $\checkmark$ & & & $\checkmark$ & & & $\checkmark$ & & $\checkmark$ & $\checkmark$ & $\checkmark$ & & & $\checkmark$ \\
\hline Tele-surgery & & $\checkmark$ & $\checkmark$ & & $\checkmark$ & & & $\checkmark$ & & & $\checkmark$ & & $\checkmark$ & $\checkmark$ & $\checkmark$ & & & $\checkmark$ \\
\hline
\end{tabular}


communication. Because the wireless nodes are battery powered, the RANET protocol has to be energy-efficient. However, it should be mentioned that in general the mobile robots or stationary network relays are equipped with rather high-capacity batteries $[10,63,70]$. Therefore, although the RANET should take measures to avoid the waste of energy because of wireless communications, this requirement is not as strict as in some other domains of ad hoc networking (e.g. wireless sensor and actor networks). In some domains such as swarm robotics, autonomous transport and military operations, it is possible that a large amount of networked robots will operate in the same location. This means that a RANET solution should be scalable: even when a large amount of network nodes are part of a single RANET, it should be able to function at the same level of quality as when just a few nodes have to be interconnected. This requires efficient approaches regarding network protocol overhead, data flooding, etc.

In terms of communication pattern, three different kinds of network topologies have to be supported: a chain network, a mesh network and a clustered network (Fig. 4). The chain network is suitable for use cases where a single robot is remotely controlled by a human operator. The mesh network is required to support use cases where robots perform their task in an autonomous manner. The clustered topology is useful when a single human operator is responsible for multiple semi-autonomous robots. On each of those topologies, both unicast, multicast and broadcast communication should be supported. Unicast is required when data has to be delivered to specific robots. This is the case in a large amount of the use cases discussed in section 2.1. Multicast communication can be required in cases where several teams are connected to the RANET, and information has to be disseminated between the team members. Example use cases are emergency networks, the indoor guidance of fire fighters and RSTA missions. Broadcast communication is required when the movement of the robots has to be coordinated without knowledge about the specific robots that are present in the immediate environment. This is the case in several applications, e.g. in the domains of autonomous transport and swarm robotics. In several use cases, the data that is exchanged over the RANET also has to be forwarded to other entities (human operators, management centers, etc.) which can geographically positioned far away from the actual robots' location. Often this objective is achieved using broadband Internet connections. Therefore it is required that RANET protocols are compatible with existing IP-based computer networks. On the other hand, to coordinate movement between a large numbers of autonomous mobile robots, it can also be required to disseminate data to all robots present within a given geographical area. Hence the RANET should provide mechanisms that support geographical networking.

In terms of data type, messaging and media streaming should both be supported. Autonomous robots often rely on the RANET for the interchange of messages with their peers. The required throughput for such applications will be rather limited, but the high mobility of robots such as UAVs imposes stringent delay requirements for coordinated multi-robot behavior. In case of tele-operation, video and audio streams are often communicated towards the operator. Such applications are much more demanding in terms of throughput. In other cases voice-over-IP streams will also be sent over the network (e.g. emergency networks, RSTA missions, etc.). This type of media 


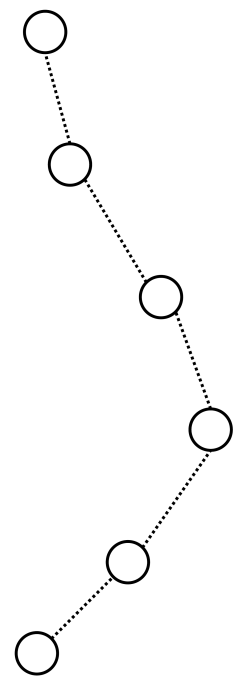

Chain network

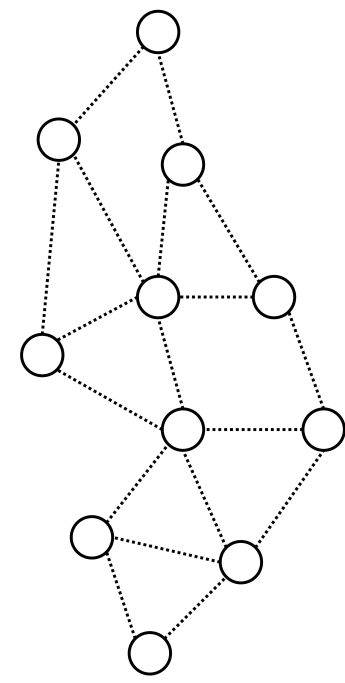

Mesh network

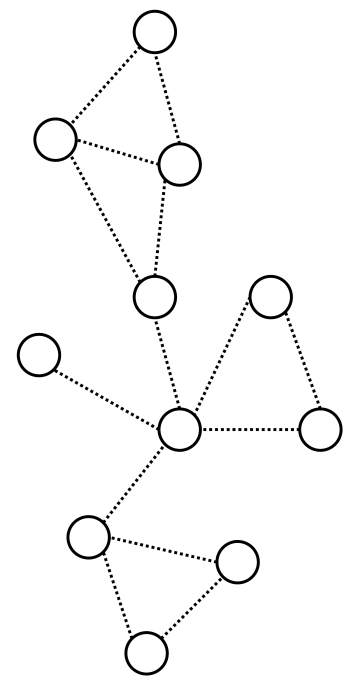

Clustered network

Fig. 4 Three different network topologies that have to be supported by a generic RANET protocol. The chain network is suitable for use cases where a single robot is remotely controlled by a human operator. The mesh network is required to support use cases where robots perform their task in an autonomous manner. The clustered topology is useful when a single human operator is responsible for multiple semiautonomous robots.

is less demanding in terms of throughput, but has very strict requirements in terms of delay. To support all these types of data with their specific requirements regarding throughput and delay, Quality of Service (QoS) mechanism are required in any generic RANET network. Besides differentiating data streams according the application requirement, these QoS mechanisms also have to support distinction according to priority within the same type. For example, in scenarios with heterogeneous robots, movement control messages of fast moving robots will be more critical than those of the slow moving robots. Although these messages are all of the same type, the QoS mechanisms of the RANET network should support prioritisation of them according to the given circumstances.

Fault tolerance is another important aspect of robotic ad hoc networking. A connected topology can become disconnected for two reasons. The first one is the dynamic nature of the topology, caused by the movement of the robots. In almost all use cases, one or more of the networked robots will be continuously moving to execute its assigned tasks. As a consequence, the network topology will continuously change, and routing paths will have to be updated frequently to avoid network disconnection. Any RANET protocol should be capable of handling these dynamic circumstances. The second reason for network disintegration is node failure. Since robots will be brought into action in harsh and dangerous environments, the probability of failure in one of the nodes is rather high. In such case, the network should also be able to automatically reconfigure itself to maintain connectivity. As indicated by the studies 
reviewed in section 2.2.4, the RANET can profit greatly from the robot's steerable mobility. The robots can be automatically positioned in such a way that maximum geographical coverage is achieved. In case of node failure, they can also be automatically repositioned to regain full network connectivity. Therefore, any solid RANET protocol should be provided with mobility control mechanisms to support RANET operations.

A last important requirement of RANET solutions is security. As explained by Zeiger et al. [71], this encompasses both operational security and data security. Operational security is related to the fact that the operation of a mobile robot like an UAV or UGV holds risks in terms of endangering or injuring persons or damaging the environment. As soon as a wireless link is incorporated into the tele-operation or control, the potential loss of the communication link must be considered. Techniques are required to prevent the robots from being a risk for the living or non-living environment. From the network point of view, this aspect of security is closely related to the requirement of fault tolerance. Data security on the other hand encloses aspects such as the prevention of misuse, intrusion or malicious interference. For instance it should be guaranteed that external parties cannot take over control of tele-operated robots. Neither should they be able to interfere with the network operation in such a way that the networked robots are no longer able to function properly. In case of military operations these requirement are quite obvious. External parties should also not be able to passively monitor the communicated data. Besides the obvious military use cases, this is also applicable for several other use cases such as mobile healthcare, urban search and rescue (where the privacy of victims and their family has to be protected), and so on. Although it can be argued that efforts on the application layer are required to provide this type of security, the RANET solution should also take action to fulfill this requirement as much as possible.

\section{Networked vehicles}

This section elaborates on different aspects of networked vehicles, similar to the survey of networked robots of the previous section. The final goal is to define the requirements of vehicular ad hoc networks (VANET). This will allow us to compare the requirements of RANET and VANET networks in section 4. Based on this comparison, we will be able to pronounce upon the feasibility of transferring techniques developed in the quite mature VANET domain into the rising domain of robotic ad hoc networks. It should be mentioned that the maturity of the VANET domain is illustrated by the wide availability of survey papers and technical standards. To keep the length of this paper within reasonable limits, we will refer to such literature as much as possible. For the domain of networked robots this kind of sources does not yet exists. Therefore we were forced to perform a thorough survey of the RANET related work ourselves. This explains the different approach in writing section 2 and section 3 of this paper. 


\subsection{Use cases}

Approximately 40 applications concerning networked cars are common in literature ${ }^{1}$. Reviewing all these applications in detail is clearly unfeasible within the constraints of this paper. For more information we therefore refer the reader to the two technical standards regarding these applications. These are provided by the Car 2 Car Communication Consortium (C2C-CC) [72] and the European Telecommunications Standards Institute (ETSI) [73]. However, for the definition of the VANET requirements in section 3.3 , the classification of the $\mathrm{C} 2 \mathrm{C}-\mathrm{CC}$ of all these applications can be very useful. The consortium was able to define six generic applications that together can support all known use cases. This classification is illustrated in Fig. 5. "Vehicle 2 Vehicle Cooperative Awareness" supports the requirement for applications to share information with each other without any persistent communication link between the vehicles. Example use cases are lane change assist, wrong way driver warning, emergency vehicle warning, crossroads collision warning, cooperative glare reduction and cooperative adaptive cruise control. "Vehicle 2 Vehicle Unicast Exchange" enables a communication link between vehicles for the exchange of information. Examples of this application are pre-crash sensing, lane merging assistance, highway platooning and instant messaging. "Vehicle 2 Vehicle Decentralized Environmental Notification" provides information about events and roadway characteristics that are probably interesting to drivers for a certain time in a certain area. Some examples are approaching traffic jam alert, slow vehicle indication, warning of incidents, road adhesion alert and notification of a broken down vehicle. "Infrastructure 2 Vehicle (One-Way)" supports the communication from roadside units (RSU) to vehicles without a persistent communication link between vehicles and RSUs. A few possibilities are contextual speed limit alerts, traffic light optimal speed advisory and wind status information at elevated road segments. "Local RSU connection" supports use cases where data between a vehicle and a RSU needs to be sent from the vehicle to the RSU or bidirectionally. This can be applicable in the case of automatic access control, free flow tolling, payment at drive-through, signal violation warning, etc. The last application, "Internet Protocol Roadside Unit Connection", supports services that are offered to the driver by servers located in the Internet. Some example use cases for this generic application are eCall, remote diagnostic, enhanced route guidance, map download, fleet management and parking management. A technical analysis of all six generic applications, containing among others the required communication techniques, is described in the C2C-CC Manifesto [72].

\footnotetext{
1 Emergency vehicle warning, slow vehicle indication, wrong way driver warning, notification of stationary or broken down vehicles, warning of accidents, approaching traffic jam alert, roadworks warning, road obstacle notification, rail crossing information, road adhesion alerts, visibility information, wind status information, contextual speed limit alerts, traffic light optimal speed advisory, enhanced route guidance, detour information, points of interest notification, parking management, loading zone information, map updates, insurance and financial services, fleet management, electronic toll collect, eCall, remote diagnostics, online car renting/sharing, stolen vehicle alert, intersection collision warning, motorcycle approaching indication, emergency electronic brake lights, signal violating warning, overtaking vehicle warning, pre-crash sensing, lane merging assistance, lane change assistance, cooperative adaptive cruise control, highway platooning, vulnerable road user protection and cooperative glare reduction
} 

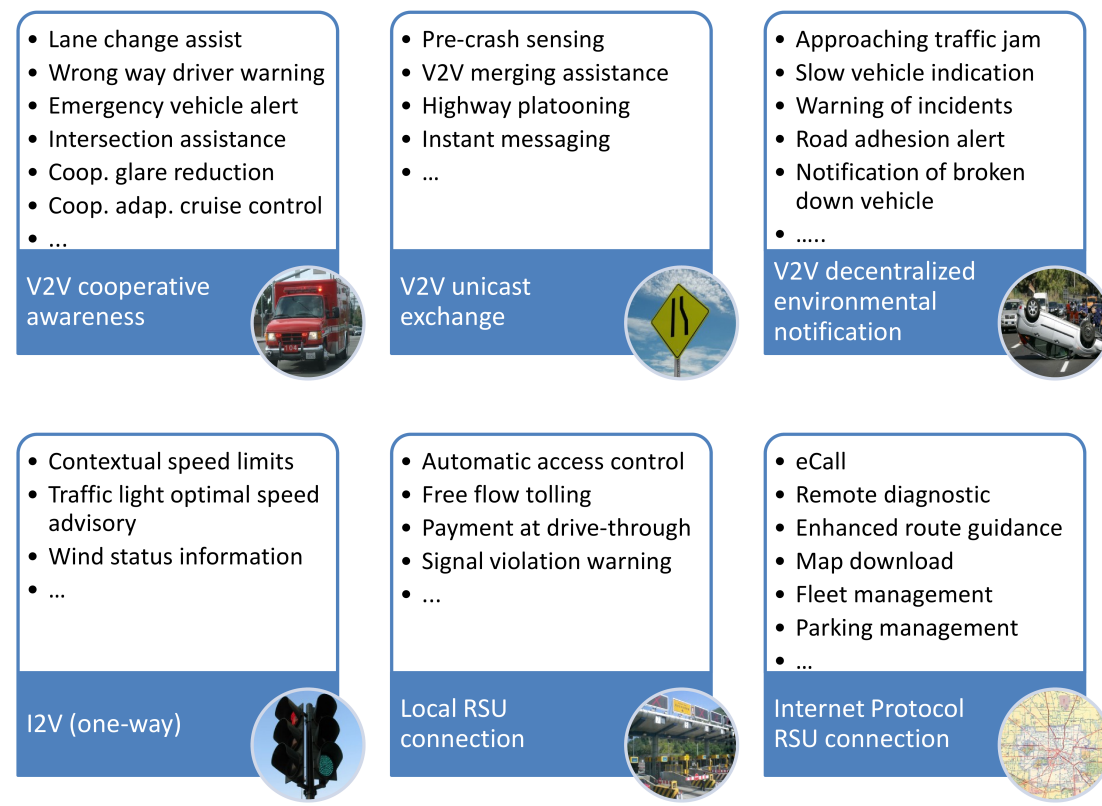

Fig. 5 Overview of uses cases where networked vehicles have been successfully applied

\subsection{Vehicular Ad Hoc Networks}

Last decade, a tremendous amount of VANET routing protocols have been presented

${ }^{2}$. Reviewing all these protocols in detail is clearly unfeasible within the constraints of this paper. We will therefore limit this discussion of the related work to a classification of the different published VANET protocols, illustrated with only a few examples. This classification is depicted in Fig. 6. For more detailed information we refer the reader to the VANET routing protocol survey papers of Blum et al. [74], Maihofer [75], Li and Wang [76], Hartenstein and Laberteaux [77], Schoch et al. [78], Bernsen and Manivannan [79], Hrizi and Filali [80], Lin et al. [81] and Chen et al. [82].

\subsubsection{IPv6 networking}

Applying IPv6 for VANET networking has some significant advantages. First of all, IP can support all types of vehicular applications, while allowing developers to rely on established networking APIs. IP can also bring legacy Internet applications (web browsing, video streaming, peer-to-peer file sharing, online gaming, etc.) to the vehicles. Since it is the de-facto standard for data exchange, IP ensures interoperability

\footnotetext{
2 MHVB, OB-VAN, R-OB-VAN, p-IVG, TO-GO, DV-CAST, GPSR, GPSR-L, MDVV, MURU, STAR, GeoDTN+Nav, VADD, SADV, CAR, MORA, CBRF, GRID, GPCR, UMB, AMB, GpsrJ+, RBVT-R, RBVT-P, GSR, A-STAR, NAR, GyTAR, SAR, CLGF and TOPO are examples of VANET protocols that were named by their authors. At least as many protocols exist that have not been named.
} 


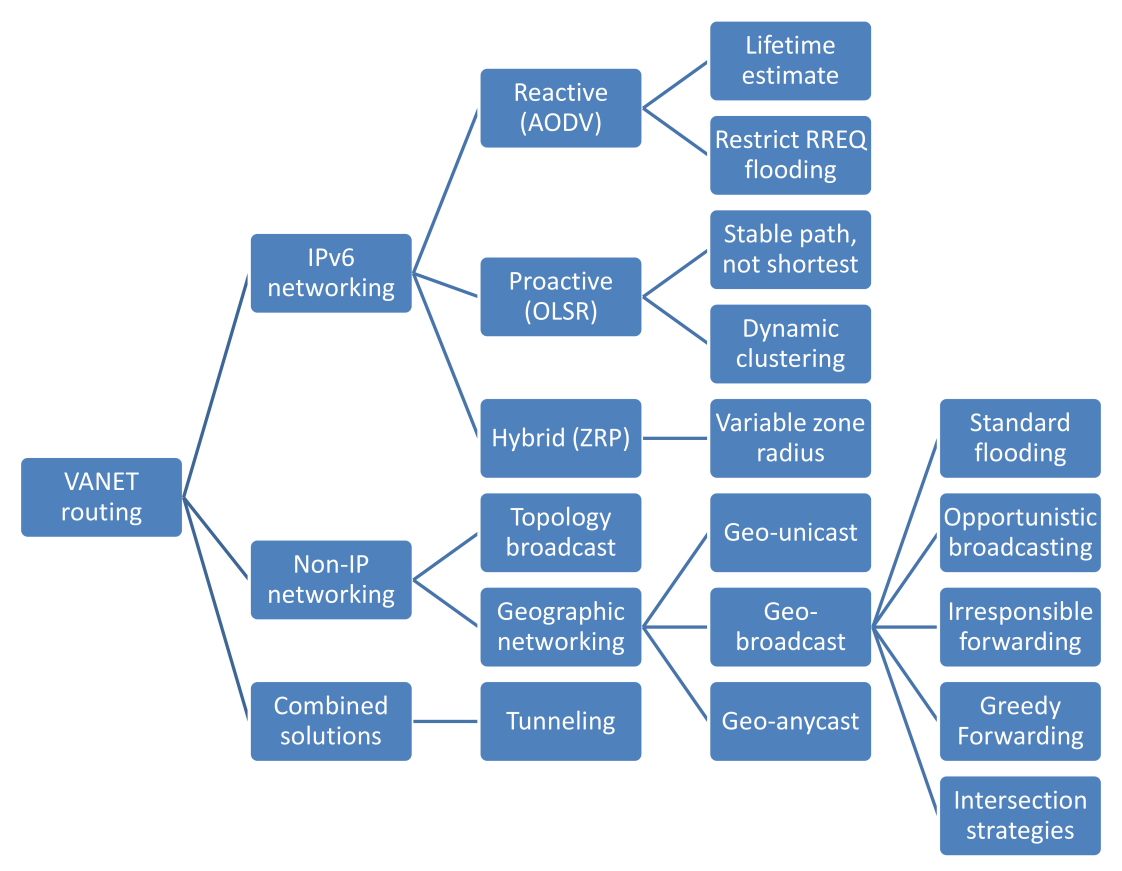

Fig. 6 Classification of different techniques known in VANET literature. This illustration only indicates the different categories, and not the amount of studies that focused on every category.

with other communication systems. Using IP, applications can run transparently over diverse underlying communication media.

The most important reason to adopt IPv6 in the VANET domain instead of the common IPv4 protocol is the fact that IPv4 does not provide a sufficient amount of available IP addresses. Because IPv4 addresses are 32 bits long, the size of the entire address space is $2^{32}$ or approx. 4.3 billion, of which the major part has already been assigned. On a global level, the Internet Assigned Numbers Authority (IANA) allocated the last available addresses on February $3^{\text {rd }}$ 2011. On a regional level, the unallocated address pool is already exhausted for one regional Internet registry (APNIC which is responsible for e.g. China and India) and it is estimated that the other regions will follow within a few years [83]. IPv6 addresses have a length of 128 bits, resulting in an address space size of $2^{128}$, completely resolving the address exhaustion problem. Other advantages of IPv6 are the provided auto-configuration capabilities and network mobility support.

A disadvantage of IPv6 is that it has no built-in notion of geographical information. This means that it does not support concepts such as geocasting where data is disseminated to vehicles within a given geographical area. Therefore, routing protocols have to rely on topology information instead of geographic information. Typically, IPv6 VANET routing protocols extend existing ad hoc protocols with techniques to improve performance and reliability. Several publications exist that focus on enhancing reactive ad hoc routing protocols such as the AODV protocol. The 
notion of link and route lifetime estimates has been introduced, based on velocity vectors and other movement information. Other studies focus on restricting the flooding of the route requests. Proactive ad hoc routing protocols such as OLSR were also extended with VANET optimizations. The MOvement Predication based Routing (MOPR) framework adjusted OLSR to prefer most stable paths instead of shortest paths. DHT-OLSR on the other hand combines OLSR with techniques from the domain of peer-to-peer networking: dynamic clustering and distributed hash table routing. The third class of existing ad hoc protocols, the hybrid routing protocols such as the Zone Routing Protocol (ZRP), has also been optimized for the VANET scenario. The Adaptive Zone Routing Protocol (AZRP) enhances the performance of ZRP with the use of a variable zone radius for every node, based on a metric called route failure rate. The Sharp Hybrid Adaptive Routing Protocol (SHARP) monitors traffic patterns and local network characteristics such as link failure rate and node degree to determine zone sizes.

\subsubsection{Non-IP networking}

Topology broadcast protocols disseminate packets from a source node to all nodes located at a specific distance, in terms of hops. WAVE Short Message Protocol (WSMP) and CALM FAST are the two most important topology broadcast protocols that aim to achieve higher repetitive broadcasting efficiency by substituting the IP protocol. WSMP is standardized by IEEE as part of the IEEE 1609.3 standard. It defines a short message header, containing information such as WSM length, version number, security info, application class, application data and transmission power, rate and channel. The length of the packet is 9 bytes plus the variable byte size of the application context data. WSMP only supports single-hop broadcasting, not multi-hop. CALM FAST is a networking protocol currently being standardized by ISO, combining networking and protocol layer functionalities. It is based on a two octet network header containing the source and destination address of the packet. The protocol is primarily designed for single-hop communications, although it supports n-hop broadcasts in the Extended CALM FAST protocol variant.

The basic idea behind geographic networking is that nodes can be addressed using geographic concepts such as locations and areas, and routing decisions can be based on inter-node distance, relative movement, etc. Depending on the destination type, several geo-routing schemes may be used. Geo-unicast routes data from a source node to a single destination node which is identified by its exact geographical location. Since this location will change over time, a position service is required that maintains a mapping in real-time between vehicle identity and exact location. Geoanycasting refers to the situation where data is routed from a source node to one random node that is located within a defined geographical broadcasting area. Geobroadcasting is used when data is routed from a source node to all nodes located within a defined geographical area. Many geonetworking protocols can be found in VANET literature, they can be clustered based on the adoption of some common techniques. The simplest technique is standard flooding. This is a broadcasting technique in which a node retransmits every newly received packet once. Another technique that is applied by many is opportunistic broadcasting, where the probability that a 
node $\mathrm{B}$ will retransmit a broadcast message sent by node $\mathrm{A}$ is dependent of the distance between $\mathrm{A}$ and $\mathrm{B}$ : the greater the distance, the higher the probability that $\mathrm{B}$ will re-broadcast. Another common technique is irresponsible forwarding, where the probability that node B will rebroadcast the broadcast message of A is dependent of the neighborhood density. Greedy forwarding lets the sender node A itself select the next node B that has to rebroadcast the message, aiming to achieve a maximum traveling distance per rebroadcast. In urban environments, intersection routing strategies are often utilized.

\subsubsection{Combined solutions}

VANETs will have to support the different functionalities provided by both the IPv6 and the non-IP solutions for the actual deployment of cooperative applications. In the GeoNet project it was researched how IPv6 connectivity can be provided in combination with the non-IP based networking protocols CALM FAST and the $\mathrm{C} 2 \mathrm{C}-\mathrm{CC}$ geographic networking protocol. It was chosen to encapsulate IPv6 packets in C2CNet packets to transport them within the GeoNet domain. Although it was proven that this approach works, tunneling makes the solutions more complex. Similar, the IEEE 1609.x family of standards will provide both WSMP and IPv6 support.

\subsection{Communication requirements}

Based on the authors' knowledge of vehicular ad hoc networks that was summarized in the previous subsections regarding use cases and VANET protocols, an exhaustive list of VANET requirements can be defined. In Table 2 the relationship between these requirements and the generic applications described in section 3.1 is illustrated.

In some cases, a vehicular ad hoc network can rely on fixed roadside infrastructure, e.g. road side units installed along highways. However, the VANET will also be required to be fully functional in many other locations where no such infrastructure will be available. A VANET should be self-organizing. In most cases, energy efficiency is of no importance since vehicles are typically equipped with high capacity batteries and generators. However, protection of vulnerable road users through cooperative awareness is becoming a topic of elevated interest. In such cases energyefficiency should at least be of concern for the mobile devices carried by the vulnerable road user. A very important requirement in VANET networking is scalability. High vehicle densities in combination with transmission ranges of approximately 1 $\mathrm{km}$ will result in scenarios where several hundreds of vehicles are within each others communication range at the same time. To maintain VANET operations, profound optimizations in terms of scalability will be essential.

In terms of communication pattern, only mesh types of networking have to be supported. The focus within VANET applications lays on messaging, not on video- or audiostreaming. This means that high throughput is not a main requirement of VANET networking. Low latency on the other hand is of the utmost importance, since many communicated messages will be related to road safety applications. These messages can be both unicasted (e.g. to enable merging-assistance or highway platooning) and 


\begin{tabular}{|c|c|c|c|c|c|c|c|c|c|c|c|c|c|c|c|c|c|c|}
\hline & 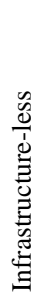 & 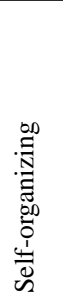 & 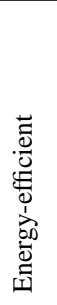 & $\begin{array}{l}\frac{0}{0} \\
\frac{\pi}{\widetilde{J}} \\
\text { wn }\end{array}$ & 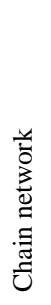 & 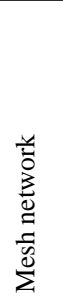 & 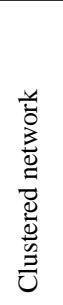 & 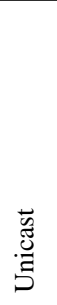 & 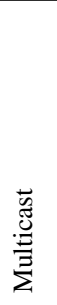 & 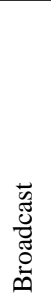 & 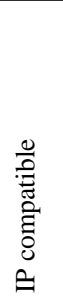 & 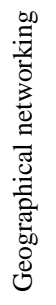 & 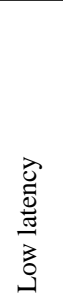 & 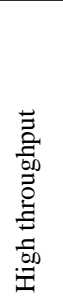 & 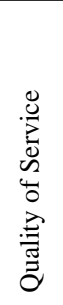 & 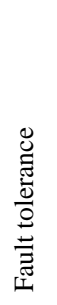 & 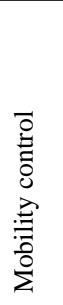 & 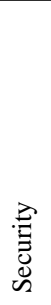 \\
\hline $\begin{array}{l}\text { V2V cooperative awareness } \\
\text { V2V unicast exchange } \\
\text { V2V environmental notification } \\
\text { I2V (one-way) } \\
\text { Local RSU connection } \\
\text { IP RSU connection }\end{array}$ & $\begin{array}{l}\checkmark \\
\checkmark \\
\checkmark\end{array}$ & $\begin{array}{l}\checkmark \\
\checkmark \\
\checkmark \\
\checkmark \\
\checkmark \\
\checkmark\end{array}$ & $\checkmark$ & $\begin{array}{l}\checkmark \\
\checkmark \\
\checkmark \\
\checkmark \\
\checkmark \\
\checkmark\end{array}$ & $\checkmark$ & $\begin{array}{l}\checkmark \\
\checkmark \\
\checkmark \\
\checkmark \\
\checkmark \\
\checkmark\end{array}$ & $\checkmark$ & $\begin{array}{l}\checkmark \\
\checkmark \\
\checkmark \\
\checkmark\end{array}$ & $\checkmark$ & $\begin{array}{l}\checkmark \\
\checkmark \\
\checkmark\end{array}$ & $\checkmark$ & $\begin{array}{l}\checkmark \\
\checkmark \\
\checkmark \\
\checkmark\end{array}$ & $\begin{array}{l}\checkmark \\
\checkmark \\
\checkmark \\
\checkmark \\
\checkmark \\
\checkmark\end{array}$ & & $\begin{array}{l}\checkmark \\
\checkmark \\
\checkmark \\
\checkmark \\
\checkmark \\
\checkmark\end{array}$ & $\begin{array}{l}\checkmark \\
\checkmark \\
\checkmark\end{array}$ & $\checkmark$ & $\begin{array}{l}\checkmark \\
\checkmark \\
\checkmark \\
\checkmark \\
\checkmark \\
\checkmark \\
\checkmark\end{array}$ \\
\hline $\begin{array}{l}\text { VANET requirements } \\
\text { RANET requirements }\end{array}$ & $\begin{array}{l}\checkmark \\
\checkmark\end{array}$ & $\begin{array}{l}\checkmark \\
\checkmark\end{array}$ & $\begin{array}{l}\checkmark \\
\checkmark\end{array}$ & $\begin{array}{l}\checkmark \\
\checkmark\end{array}$ & $\begin{array}{l}\checkmark \\
\checkmark\end{array}$ & $\begin{array}{l}\checkmark \\
\checkmark\end{array}$ & $\begin{array}{l}\checkmark \\
\checkmark\end{array}$ & $\begin{array}{l}\checkmark \\
\checkmark\end{array}$ & $\begin{array}{l}\checkmark \\
\checkmark\end{array}$ & $\begin{array}{l}\checkmark \\
\checkmark\end{array}$ & $\begin{array}{l}\checkmark \\
\checkmark\end{array}$ & $\begin{array}{l}\checkmark \\
\checkmark\end{array}$ & $\begin{array}{l}\checkmark \\
\checkmark\end{array}$ & $\checkmark$ & $\begin{array}{l}\checkmark \\
\checkmark\end{array}$ & $\begin{array}{l}\checkmark \\
\checkmark\end{array}$ & $\begin{array}{l}\checkmark \\
\checkmark\end{array}$ & $\begin{array}{l}\checkmark \\
\checkmark\end{array}$ \\
\hline
\end{tabular}


broadcasted (e.g. to warn oncoming traffic about incidents, slippery spots, slow vehicles, and so on). Compatibility with existing IP-based computer networks is required by all applications relying on Internet-based services. Examples are eCall, remote diagnostic, enhanced route guidance and fleet management. Geographical networking is a major demand of VANET applications. When disseminating information about vehicles or road conditions towards oncoming traffic, it is absolutely required that the destination of a message can be defined on a geographical basis. QoS mechanisms should also be in place to differentiate between critical and non-critical information. Fault tolerance is of less importance for most safety related applications since those focus more on broadcasting information over a given area instead of creating multihop communication paths. This relieves any sensitivities regarding node failure or dynamic topologies. However, for some comfort applications, a multi-hop connection from the vehicle to the Internet-connected RSU will be utilized (such as instant messaging and enhanced route guidance). In that case fault tolerance can be required. Mobility control mechanisms are not required in the VANET environment. Security on the other hand is very important, with the prevention of fake message injection by attackers being one of the biggest challenges.

\section{Comparison of RANET and VANET domain}

As mentioned in the introduction, the goal of this paper is to assess if the results of the many research efforts that focused on the VANET domain could be transfered to the RANET domain. If possible, this could lead to significant acceleration of the technical developments in the domain of networked robots. In the previous two sections, both domains were studied in detail, and an exhaustive list of requirements was composed for each of them. By comparing these requirements, it is now possible to estimate the degree of similarity between both domains. Based on this analysis, recommendations can be formulated regarding the approach towards future RANET research

\subsection{Similarities and differences}

As illustrated in the bottom part of Table 2, both domains have almost equal requirements. According to this global overview, the only difference is that VANET protocols do no intend to support flows with high throughput demands (such as high definition video). All other requirements are shared between both domains. This clearly indicates the resemblance of both domains, and encourages to experiment with crossfertilization between them in future research. To be able to define an action plan for such work in the next section, it can be interesting to know which requirements have received more attention in both domains, since this gives an indication of the strengths and weaknesses of the existing solutions. As can be observed in Table 1, some RANET requirements where applicable for almost all use cases, while others were less common. These key requirements are infrastructure-less operation, selforganization, energy-efficiency, unicast support, IP compatibility, low latency, high 
throughput, quality of service and security. When looking back at the survey of existing RANET protocols given in section 2.2, it can be confirmed that these requirements were in general targeted in all developed solutions. It should also be mentioned that the requirement of mobility control, although not a requirement for all use cases, has also received a large amount of attention as described in section 2.2.4. In the domain of vehicular ad hoc networking, the list of key requirements is somewhat different. Self-organization, scalability, mesh networking, broadcasting, geographical networking, low latency, quality of service and security were required in almost all cases. When taking the survey of existing VANET protocols of section 3.2 into account, it can be confirmed that these requirements were in general targeted in most developed solutions.

\subsection{Recommendations regarding future RANET research}

Based on these observations we can now define recommendations for future RANET development. The first required step is to analyze which of the existing RANET ad hoc networking solutions achieves best results in terms of the key requirements for robotic ad hoc networking. As indicated in section 2.2, original MANET protocols have already been studied thoroughly in literature, and they are not suitable as RANET protocols. A few adapted MANET protocols and novel RANET showcased were found which present interesting ideas. However, in most cases performance measurements were inadequate, and mobility levels during the experiments were too low. Therefore the first task at hand is to execute a thorough performance analysis of these existing solutions, relying on adequate experimentation scenarios. Ideally, these tests will be performed both on simulators and actual testbeds for networked robots. Such tools are already in place. For simulations, many authors rely on an integrated simulation platform that combines a robot simulator (e.g. Microsoft robotics studio, Webots, USARSim, Player/Stage or Gazebo) with a wireless network simulator (e.g. GloMoSim, NS-2, NS-3, OPNET) [8, 84, 85]. Other studies presented real life testbeds [86-88]. Reusing such existing tools in future research efforts seems sensible.

Based on these performance analysis, a suitable ad hoc networking basis for a generic RANET protocol can be identified. Then a suitable movement control algorithm has to be added to the solution. A large amount of such algorithms is already available as indicated in section 2.2.4. Therefore it is advised to combine the chosen ad hoc networking protocol with some of these available control algorithms, and analyze which combinations performs best.

After this step, the best possible RANET solution based on the current state of the art in this domain will be available. For some RANET requirements, it will however be not yet optimized. Because several of these requirements are key features of VANET protocols, the next step is to introduce optimization techniques used by these VANET protocols into the generic RANET solution. Topics where most profit can be gained from this approach are scalability, mesh network routing, geographical networking and broadcasting. In theory, the generic RANET solution should already target requirements such as self-organization, low latency, QoS and security in a satis- 
factory manner. However, if these aspects are found to be not mature enough, VANET solutions can also be very valuable inspiration sources. From a technical point of view, transferring techniques from VANET to RANET implementations should be relatively straightforward since VANET research typically relies on testbeds and simulators that integrate a vehicle simulator with a wireless network simulator [89-91]. This is similar to the RANET approach.

Finally, to provide solid coverage of all RANET requirements, attention should be given to those requirements that are not a key requirement in the RANET or the VANET domain. First of all, it should be validated if these requirements are already met in a sufficient manner. If not, specific optimizations have to be introduced in the generic RANET protocol. These can be inspired by publications that especially focused on one of these requirements in the context of very specific uses cases, or can be developed from scratch. This task marks the final step of the development of a single RANET solution that can be applied in all very heterogeneous use cases that are known in the domain of networked robots.

\section{Conclusions}

In this study, a thorough review was given of the state of art in the domains of robotic and vehicular ad hoc networking. Based on this information, an exhaustive list of requirements was given for both types of networking protocols. In general, the most significant difference lies in the fact that VANET protocols are more oriented towards low throughput messaging, while RANET protocols have to support high throughput media streaming as well. All other requirements were valid for both protocols. From this observation it can be concluded that cross-fertilization between them is a very appealing approach for future RANET research. Such work should consider the fact that existing solutions for both types of protocols target different key requirements. Therefore a working plan was defined for the design and development of a generic RANET solution that can be applied in the large amount of heterogeneous use cases that are known in the domain of networked robots. First a thorough performance analysis of current state of the art RANET protocols has to be performed to identify the most suitable basis for ad hoc networking. Then combinations of this starting point have to be combined with known movement control protocols to point out the optimal solution based on the current RANET state of the art. The next step is to transfer techniques from the VANET domain to the generic RANET solution to cover requirements that need further attention. Finally, missing links in the generic RANET protocol have to be identified and tackled with novel developments.

\section{References}

1. Jung, J.H., Park, S. \& Kim, S.-L. (2010). Multi-robot path finding with wireless multihop communications. IEEE Communications magazine, 48(7), 126-132

2. Sugiyama, H., Tsujioka, T. \& Murata, M. (2009). Integrated Operations of Multi-Robot Rescue System with Ad Hoc Networking. In: Proceedings of Wireless Communications, Vehicular Technology, Information Theory and Aerospace \& Electronic Systems Technology. Germany 
3. Birk., A., Schwertfeger, S. \& Pathak, K. (2009). A networking framework for teleoperation in safety, security and rescue robotics. IEEE Wireless communications, 16(1), 6-13

4. Zhang, Y., Zeng, L., Li, Y. \& Liu, Q. (2009). Multi-robot formation control using leader-follower for MANET. In: Proceedings of the 2009 IEEE International Conference on Robotics and Biomimetics. China.

5. Royer, E.M. \& Toh, C.-K. (1999). A review of current routing protocols for ad hoc mobile wireless networks. IEEE personal communications, 6(2), 46-55.

6. A. Sanfeliu, N. Hagita \& Saffiotti, A. (2008). Network robot systems. Robotics and autonomous systems, 56(10), 793-797

7. Murphy, R.R. (2004). Trial by fire - Activities of the rescue robots at the World Trace Center from 11-21 September 2001. IEEE Robotics \& automation magazine, 11(3), 50-61

8. Sweeney, J.D., Grupen, R. \& Shenoy, P. (2004). Active QoS flow maintenance in controlled mobile networks. In: Proceedings of the fourth international symposium on robotics and automation (ISRA). Mexico

9. Antonelli, G., Arrichiello, F., Chiaverini, S., Contrafatto, S. \& Setola, R. (2007). Use of a robot platoon to implement mobile ad-hoc network in rescue scenario - preliminary experimental results. In: Proceedings of the 2007 IEEE international workshop on safety, security and rescue robotics. Italy

10. Matsuno, F., Kamegawa, T., Sato, N., Hatayama, M., Mizumoto, H., Oh-hara, S., et al. (2010). Rescue Robot Systems - Development of high-functionality multiple robot system and robust/scalable information infrastructure. In: Proceedings of the 2010 IEEE workshop on advanced robotics and its social impacts (ARSO). South Korea.

11. Correll, N., Bachrach, J., Vickery, D. \& Rus, D. (2009). Ad-hoc wireless network coverage with networked robots that cannot localize. In: Proceedings of the 2009 IEEE International conference on robotics and automation. Japan.

12. Ollero, A., Alcazar, J., Cuesta, F., Lopez-Pichaco, F. \& Nogales, C. (2003). Helicopter teleoperation for aerial monitoring in the COMETS multi-UAV system. In: Proceedings of the 3rd LARP workshop on service, assistive and personal robots. Spain

13. Penders, J., Alboul, L., Witkowski, U., Naghsh, A. Saez-Pons, J., Herbrechtsmeier, S., et al. (2011). A robot swarm assisting a human fire-fighter. Advanced robotics, 25(1-2), 93-117

14. Baber, J., Kolodko, J., Noel, T., Parent, M. \& Vlacic, L. (2005). Cooperative autonomous driving Intelligent vehicles sharing city roads. IEEE robotics \& automation magazine, 12(1), 44-49

15. Nagel, R., Eichler, S. \& Eberspcher, J. (2007). Intelligent wireless communication for future autonomous and cognitive automobiles. In: Proceedings of the 2007 Intelligent Vehicles Symposium. Turkey

16. Xiong, N., Vasilakos, A.V., Yang, L.T., Pedrycz, W., Zhang, Y. \& Li, Y. (2010). A resilient and scalable flocking scheme in autonomous vehicular networks. Mobile networks and applications, 15(1), 126-136 17. Milanes, V., Alonso, J., Bouraoui, L. \& Ploeg, J. (2011). Cooperative maneuvering in close environments among cybercars and dual-mode cars. IEEE Transactions on intelligent transportation systems, $12(1), 15-24$

18. Sanders, G. (2004). Boeing technical experts check the feasibility of Personal Air Vehicles. Boeing Frontiers. http://www.boeing.com/news/frontiers/archive/2004/july/ts_sf14.html. Accessed 22 September 2011.

19. Thrun, S., Thayer, S., Whittaker, W., Baker, C., Burgard, W., Ferguson, D., et al. (2004). Autonomous exploration and mapping of abandoned mines - Software architecture of an autonomous robotic system. IEEE robotics \& automation magazine, 11(4), 79-91

20. Murphy, R.R., Kravitz, J., Stover, S.L. \& Shoureshi, R. (2009). Mobile robots in mine rescue and recovery. IEEE Robotics \& automation magazine, 16(2), 91-103

21. Weiss, M.D., Peak, J. \& Schwengler, T. (2008). A statistical radio range model for a robot MANET in a subterranean mine. IEEE Transactions on vehicular technology, 57(5), 2658-2666

22. Parker, L.E. (1998). ALLIANCE: An architecture for fault tolerant multirobot cooperation. IEEE Transactions on Robotics and Automation, 14(2), 220-240.

23. Ibach, P., Milanovic, N., Richlilng, J., Stantchev, V., Wiesner, A. \& Malek, M. (2005). CERO: CE robots community. IEEE Proceedings Software, 152(5), 210-214.

24. Kovacs, T., Pasztor, A. \& Istenes, Z. (2009). Connectivity in a wireless network of mobile robots doing a searching and collecting task. In: Proceedings of the 5th International symposium on applied computational intelligence and informatics. Romania.

25. Steels, L. (1990). Cooperation between distributed agents through self-organization. In: Proceedings of the first European workshop on modeling autonomous agents in a multi-agent world. UK. 
26. Stilwell, D. \& Bay, J. (1993). Towards the development of a material transport system using swarms of ant-like robots. In: Proceedings of the IEEE international conference on robotics and automation. USA.

27. Erickson, J.K. (2006). Living the dream - An overview of the Mars exploration project. IEEE Robotics \& automation magazine, 13(2), 12-18

28. Rooker, M.N. \& Birk, A. (2007). Multi-robot exploration under the constraints of wireless networking. Control Engineering Practice, 15(4), 435-445

29. Toung, J., Gilstrap, R. \& Freeman, K. (2006). A split implementation of the dynamic source routing protocol for lunar/planetary surface communications. In: Proceedings of the 2006 IEEE aerospace conference. USA.

30. Bevilacqua, R., Hall, J.S., Horning, J. \& Romano, M. (2009). Ad hoc wireless networking and shared computation for autonomous multirobot systems. Journal of aerospace computing information and communication, 6(5), 328-353

31. Pompili, D. \& Akyildiz, I.F. (2009). Overview of networking protocols for underwater wireless communications. IEEE Communications magazine, 47(1), 97-102

32. Vasilescu, I., Detweiler, C., Doniec, M., Gurdan, D., Sosnowski, S., Stumpf, J., et al. (2010). AMOUR $\mathrm{V}$ : a hovering energy efficient underwater robot capable of dynamic payloads. International journal of robotics research, 29(5), 547-570

33. Benton, C., Kenney, J., Nitzel, R., Blidberg, D.R., Chappell, S. \& Mupparapu, S. (2004). Autonomous undersea systems network (ausnet) - Protocols to support ad-hoc AUV communications. In: Proceedings of AUV2004: A Workshop on Multiple Autonomous Underwater Vehicle Operations. USA.

34. Clark, J. \& Fierro, R. (2007). Mobile robotic sensors for perimeter detection and tracking. ISA Transactions, 46(1), 3-13

35. Kingston, D., Beard, R.W. \& Holt, R.S. (2008). Decentralized perimeter surveillance using a team of UAVs. IEEE Transactions on robotics, 24(6), 1394-1404

36. Calkins, D. (2011). An overview of RoboGames. IEEE robotics \& automation magazine, 18(1), 14-15

37. Behnke, S. \& Stckler, J. (2008). Hierarchical reactive control for humanoid soccer robots. International journal of humanoid robotics, 5(3), 375-396

38. Weitzenfeld, A., Martinez-Gomez, L., Francois, J.P., Levin-Pick, A., Obraczka, K. \& Boice, J. (2006). Multi-robot systems: extending Robocup small-size architecture with local vision and ad-hoc networking. In: Proceedings of the 2006 IEEE 3rd latin American robotics symposium. Chile

39. Fiene, J. (2010). The robockey cup - A look at mechatronics education in 2009. IEEE robotics \& automation magazine, 17(3), 78-82

40. Ben-Asher, Y., Feldman, S., Gurfil, P. \& Feldman, M. (2008). Distributed decision and control for cooperative UAVs using ad hoc communication. IEEE Transactions on control systems technology, 16(3), 511-516.

41. Luu, B.B., O’Brien, B.J., Baran, D.G. \& Hardy, R.L. (2007). A soldier-robot ad hoc network. In: Proceedings of the fifth annual IEEE international conference on pervasive computing and communications workshop (PerComW). USA.

42. Nguyen, H.G., Pezeshkian, N., Raymond, M., Gupta, A. \& Spector, J.M. (2003). Autonomous communication relays for tactical robots. In: Proceedings of the 11th international conference on advanced robotics. Portugal

43. Hsieh, M.A., Cowley, A., Keller, J.F., Chaimowicz, L., Grocholsky, V.K., Kumar, V., et al. (2007). Adaptive teams of autonomous aerial and ground robots for situational awareness. Journal of field robotics, 24(11-12), 991-1014

44. Sariel, S., Balch, T. \& Erdogan, N. (2008). Naval mine countermeasure missions - A distributed, incremental multirobot task selection scheme. IEEE robotics \& automation magazine, 15(1), 45-52

45. Lindemuth, M., Murphy, R., Steimle, E., Armitage, W., Dreger, K., Elliot, T., et al. (2011). Sea robotassisted inspection. IEEE robotics \& automation magazine, 18(2), 96-107

46. Pezeshkian, N., Nguyen, H.G. \& Burmeister, A. (2007). Unmanned ground vehicle radio relay deployment system for non-line-of-sight operations. In: Proceedings of the 13th IASTED international conference on robotics \& applications. Germany

47. Nguyen, H.G., Kogut, G., Barua, R., Burmeister, A., Pezeshkian, N., Powell, D., et al. (2004). A Segway RMP-based robotic transport system. In: Proceedings of conference on mobile robots XVII. USA

48. Jiang, J.-R., Lai, Y.-L. \& Deng, F.-C. (2011). Mobile robot coordination and navigation with directional antennas in positionless wireless sensor networks. International journal of ad hoc and ubiquitous computing, 7(4), 272-280 
49. Garawi, S., Istepanian, R.S.H. \& Abu-Rgheff M.A. (2006). 3G wireless communications for mobile robotic tele-ultrasonography systems. IEEE Communications magazine, 44(4), 91-96

50. Takeuchi, R., Harada, H., Masuda, K., Ota, G.i., Yokoi, M., Teramura, N., et al. (2008). Field testing of a remote controlled robotic tele-echo system in an ambulance using broadband mobile communication technology. Journal of medical systems, 32(3), 235-242.

51. Petelin, J.B., Nelson, M.E. \& Goodman, J. (2007). Deployment and early experience with remotepresence patient care in a community hospital. Surgical endoscopy and other interventional techniques, 21(1), 53-56

52. Harnett, B.M., Doarn, C.R., Rosen, J., Hannaford, B. \& Broderick, T.J. (2008). Evaluation of unmanned airborne vehicles and mobile robotic telesurgery in an extreme environment. Telemedicine journal and E-health, 14(6), 539-544

53. Wang, Z., Liu, L. \& Zhou, M. (2005). Protocols and applications of ad-hoc robot wireless communication networks: an overview. International journal of intelligent control and systems, 10(4), 296-303.

54. Perkins, C. \& Belding-Royer, E. (2003). RFC 3561: Ad hoc On-Demand Distance Vector (AODV) routing. Internet Engineering Task Force.

55. Pohjola, M., Nethi, S. \& Jantti, R. (2009). Wireless control of a multihop mobile robot squad. IEE wireless communications, 16(1), 14-20

56. Kudelski, M., Gadomska-Kudelska, M. \& Pacut, A. (2008). Geographical cells routing in ad-hoc networks of mobile robots. In: Proceedings of the 14th IEEE mediterranean electrotechnical conference (MELECON). France.

57. Zeiger, F., Kraemer, N. \& Schilling, K. (2008). Commanding mobile robots via wireless ad-hoc networks - a comparison of four ad-hoc routing protocol implementations. In: Proceedings of the 2008 IEEE international conference on robotics and automation. USA

58. Johnson, D., Hu, Y. \& Maltz, D. (2007). RFC 4728: The Dynamic Source Routing protocol (DSR) for mobile ad hoc networks for IPv4. Internet Engineering Task Force.

59. Clausen, T. \& Jacquet, T. (2003). RFC 3626: Optimized Link State Routing Protocol (OLSR). Internet Engineering Task Force.

60. Zeiger, F., Kraemer, N., Sauer, M. \& Schilling, K. (2009). Mobile robot teleoperation via wireless multihop networks - parameter tuning of protocols and real world application scenarios. Lecture notes in electrical engineering, 37(3), 139-152

61. Das, S., Hu, Y.C., Lee, C.S.G. \& Lu, Y.-H. (2007). Mobility-aware ad hoc routing protocols for networking mobile robot teams. Journal of communications and networks, 9(3), 296-311

62. Tiderko, A., Bachran, T., Hoeller, F. \& Schulz, D. (2008). Rose - A framework for multicast communication via unreliable networks in multi-robot systems. Robotics and autonomous systems, 56(12), $1017-1026$

63. Pezeshkian, N., Nguyen, H.G., Burmeister, A., Holz, K. \& Hart, A. (2010). Automatic payload deployment system. In: Proceedings of conference on unmanned systems technology XII. USA

64. Dixon, C. \& Frew, E.W. (2009). Maintaining optimal communication chains in robotic sensor networks using mobility control. Mobile networks \& applications, 14(3), 281-291

65. Basu, P. \& Redi,J. (2004). movement control algorithms for realization of fault-tolerant ad hoc robot networks. IEEE Network, 18(4), 36-44.

66. Das, S., Liu, H., Nayak, A. \& Stojmenovic, I. (2009). A localized algorithm for bi-connectivity of connected mobile robots. Telecommunications systems, 40(3-4),129-140

67. Liu, H., Chu, X., Leung, Y.-W. \& Du, R. (2010). Simple movement control algorithm for biconnectivity in robotic sensor networks. IEEE journal on selected areas in communications, 28(7), 994-1005

68. Ulam, P. \& Arkin, R.C. (2004). When good comms go bad: communications recovery for multi-robot teams. In: Proceedings of the 2004 IEEE International Conference on Robotics \& Automation. USA.

69. Lee, D.-J. \& Mark, R. (2010). Decentralized control of unmanned aerial robots for wireless airborne communication networks. International journal of advanced robotic systems, 7(3), 191-200

70. Ben-Tzvi, P., Goldenberg, A.A. \& Zu, J.W. (2010). Articulated hybrid mobile robot mechanism with compounded mobility and manipulation and on-board wireless sensor/actuator control interfaces. Mechatronics, 20(6), 627-639

71. Zeiger, F., Kraemer, N., Sauer, M. \& Schilling, K. (2008). Challenges in realizing ad-hoc networks based on wireless LAN with mobile robots. In: Proceedings of the 6th International symposium on modelling and optimization in mobile, ad hoc and wireless networks (WiOPT). Germany

72. Car 2 Car Communication Consortium (2007). C2C-CC Manifesto. http://www.car-to-car.org. Accessed 27 September 2011. 
73. European Telecommunications Standards Institute (2009). TR 102638 ; Intelligent Transport Systems; Vehicular Communications; Basic set of applications; Definitions. http://www.etsi.org. Accessed 27 September 2011.

74. Blum, J.J., Eskandarian, A. \& Hoffman, L.J. (2004). Challenges of intervehicle ad hoc networks. IEEE Transactions on intelligent transportation systems. 5(4), 347-351

75. Maihofer, C. (2004). A survey of geocast routing protocols. IEEE Communications surveys and tutorials, $6(2), 32-42$

76. Li, F. \& Wang, Y. (2007). Routing in vehicular ad hoc networks: a survey. IEEE Vehicular technology magazine, 2(2), 12-22

77. Hartenstein, H. \& Laberteaux, K.P. (2008). A tutorial survey on vehicular ad hoc networks. IEEE Communications magazine, 46(4), 164-171

78. Schoch, E., Kargl, F., Weber, M. \& Leinmuller, T. (2008). Communication patterns in VANETs. IEEE Communications magazine, 46(11), 119-125

79. Bernsen, J. \& Manivannan, D. (2009). Unicast routing protocols for vehicular ad hoc networks: a critical comparison and classification. Pervasive and mobile computing, 5(1), 1-18

80. Hrizi, F. \& Filali, F. (2009). On congestion-aware broadcasting in V2X networks. In: Proceedings of the international conference on ultra modern telecommunications \& workshops. Rusia.

81. Lin, Y.-W., Chen, Y.-S. \& Lee, S.-L. (2010). Routing protocols in vehicular ad hoc networks: a survey and future perspectives. Journal of information science and engineering, 26(3), 913-932

82. Chen, W., Guha, R.K., Kwon, T.J., Lee, J. \& Hsu, Y.-Y. (2011). A survey and challenges in routing and data dissemination in vehicular ad hoc networks. Wireless communication and mobile computing, 11(7), 787-795

83. Huston, G. IPv4 address report. http://www.potaroo.net/tools/ipv4/index.html. Accessed 27 September 2011.

84. Jackson, J. (2007). Microsoft robotics studio: a technical introduction - Standardizing robotic coordination and control. IEEE robotics \& automation magazine, 14(4), 82-87

85. Fink, J., Collins, T., Kumar, V., Mostofi, Y., Baras, J., et al. (2009). A simulation environment for modeling and development of algorithms for ensembles of mobile microsystems. In: Proceedings of the SPIE conference on micro- and nanotechnology sensors, systems, and applications. USA.

86. Chung, T., Cremean, L., Dunbar, W.B., Jin, Z., Klavins, E., Moore, D., et al. (2002). A platform for cooperative and coordinated control of multiple vehicles. In: Proceedings of the 3rd conference on cooperative control and optimization. USA

87. Michael, N., Fink, J. \& Kumar, V. (2008). Experimental testbed for large multirobot teams - verification and validation. IEEE robotics and automation magazine, 15(1), 53-61

88. Wilson, C.G. \& Roppel, T. (2009). Low-cost wireless mobile ad-hoc network robotic testbed. In: Proceedings of the 5th International conference on testbeds and research infrastructures for the development of networks \& communities (Tridentcom). USA.

89. Piorkowski, M., Raya, M., Lezama Lugo, A., Papadimitratos, P., Grossglauser, M. \& Hubaux, J.-P. (2008). TraNS: realistic joint traffic and network simulator for VANETs. ACM SIGMOBILE Mobile computing and communications review, 12(1), 31-33.

90. Liu, B., Khorashadi, B., Du, H., Ghosal, D., Chuah, C.-N. \& Zhang, M. (2009). VGSim: an integrated networking and microscopic vehicular mobility simulation platform. IEEE Communications Magazine, 47(5), 131-141

91. Maneros, J., Rondinone, M., Gonzalez, A., Bauza, R. \& Krajzewicz, D. (2009). iTETRIS platform architecture for the intergration of cooperative traffic and wireless simulations. In: Proceedings of the 9 th international conference on ITS telecommunications. France. 